\title{
Bubbles with constant mean curvature, and almost constant mean curvature, in the hyperbolic space
}

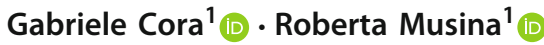

Received: 14 September 2020 / Accepted: 31 January 2021 / Published online: 16 September 2021

(c) The Author(s) 2021

\section{Abstract}

Given a constant $k>1$, let $Z$ be the family of round spheres of radius $\operatorname{artanh}\left(k^{-1}\right)$ in the hyperbolic space $\mathbb{H}^{3}$, so that any sphere in $Z$ has mean curvature $k$. We prove a crucial nondegeneracy result involving the manifold $Z$. As an application, we provide sufficient conditions on a prescribed function $\phi$ on $\mathbb{H}^{3}$, which ensure the existence of a $\mathcal{C}^{1}$-curve, parametrized by $\varepsilon \approx 0$, of embedded spheres in $\mathbb{H}^{3}$ having mean curvature $k+\varepsilon \phi$ at each point.

Mathematics Subject Classification 53A10 · 35R01 · 53C21

\section{Introduction}

Let $K$ be a given function on the hyperbolic space $\mathbb{H}^{3}$. The $K$-bubble problem consists in finding a $K$-bubble, which is an immersed surface $u: \mathbb{S}^{2} \rightarrow \mathbb{H}^{3}$ having mean curvature $K$ at each point. Besides its independent interest, the significance of the $K$-bubble problem is due to its connection with the Plateau problem for disk-type parametric surfaces having prescribed mean curvature $K$ and contour $\Gamma$, see for instance [1,13]. In the Euclidean case, the impact of $K$-bubbles on nonexistence and lack of compactness phenomena in the Plateau problem has been investigated in $[5,8,9]$.

To look for $K$-bubbles in the hyperbolic setting one can model $\mathbb{H}^{3}$ via the Poincaré upper half-space $\left(\mathbb{R}_{+}^{3}, p_{3}^{-2} \delta_{h j}\right)$ and consider the elliptic system

$$
\Delta u-2 u_{3}^{-1} G(\nabla u)=2 u_{3}^{-1} K(u) \partial_{x} u \wedge \partial_{y} u
$$

Communicated by Manuel del Pino.

Gabriele Cora

gabriele.cora@uniud.it

Roberta Musina

roberta.musina@uniud.it

1 Dipartimento di Scienze Matematiche, Informatiche e Fisiche, Università di Udine, Udine, Italy 
for functions $u=\left(u_{1}, u_{2}, u_{3}\right) \in \mathcal{C}^{2}\left(\mathbb{S}^{2}, \mathbb{H}^{3}\right)$. Here we used the stereographic projection to introduce local coordinates on $\mathbb{S}^{2} \equiv \mathbb{R}^{2} \cup\{\infty\}$ and put

$$
G_{\ell}(\nabla u):=\nabla u_{3} \cdot \nabla u_{\ell}-\frac{1}{2}|\nabla u|^{2} \delta_{\ell 3}=-\frac{1}{2} u_{3} \sum_{h, j=1}^{3} \Gamma_{h j}^{\ell}(u) \nabla u_{h} \cdot \nabla u_{j}, \quad \ell=1,2,3,
$$

where $\Gamma_{h j}^{\ell}$ are the Christoffel symbols. Any nonconstant solution $u$ to (1.1) is a generalized $K$ bubble in $\mathbb{H}^{3}$ (see Lemma A.2 in the Appendix and [14, Chapter 2]), that is, $u$ is a conformal parametrization of a surface having mean curvature $K(u)$, apart from a finite number of branch points. Once found a solution to (1.1), the next step should concern the study of the geometric regularity of the surface $u$, which might have self-intersections and branch points.

A remarkable feature of (1.1) is its variational structure, which means that its solutions are critical points of a certain energy functional $E$, see the Appendix for details. Because of their underlying geometrical meaning, both (1.1) and $E$ are invariant with respect to the action of Möbius transformations. This produces some lack of compactness phenomena, similar to those observed in the largely studied $K$-bubble problem, raised by S.T. Yau [22], for surfaces in $\mathbb{R}^{3}$ (see for instance $[7,10,12,20]$ and references therein; see also the pioneering paper [23] by Ye and [3,6,19,21] for related problems). However, the hyperbolic $K$-bubble problem is definitively more challenging, due to the homogeneity properties that characterize the hyperbolic-area and the hyperbolic-volume functionals.

The main differences between the Euclidean and the hyperbolic case are already evident when the prescribed curvature is a constant $k>0$ (the case $k<0$ is recovered by a change of orientation). Any round sphere of radius $1 / k$ in $\mathbb{R}^{3}$ can be parameterized by an embedded $k$-bubble, which minimizes the energy functional

$$
E_{\text {Eucl }}(u)=\frac{1}{2} \int_{\mathbb{R}^{2}}|\nabla u|^{2} d z+\frac{2 k}{3} \int_{\mathbb{R}^{2}} u \cdot \partial_{x} u \wedge \partial_{y} u d z
$$

on the Nehari manifold $\left\{u \neq\right.$ const. $\left.\mid E_{\text {Eucl }}^{\prime}(u) u=0\right\}$, see [7, Remark 2.6]. In contrast, no immersed hyperbolic $k$-bubble exists if $k \in(0,1]$, see for instance [16, Theorem 10.1.3]. If $k>1$, then any sphere in $\mathbb{H}^{3}$ of radius

$$
\rho_{k}:=\operatorname{artanh} \frac{1}{k}=\frac{1}{2} \ln \frac{k+1}{k-1}
$$

can be parameterized by an embedding $U: \mathbb{S}^{2} \rightarrow \mathbb{H}^{3}$, which solves

$$
\Delta u-2 u_{3}^{-1} G(\nabla u)=2 u_{3}^{-1} k \partial_{x} u \wedge \partial_{y} u \text { on } \mathbb{R}^{2},
$$

and which is a critical point of the energy functional

$$
E_{\text {hyp }}(u)=\frac{1}{2} \int_{\mathbb{R}^{2}} u_{3}^{-2}|\nabla u|^{2} d z-k \int_{\mathbb{R}^{2}} u_{3}^{-2} e_{3} \cdot \partial_{x} u \wedge \partial_{y} u d z .
$$

As in the Euclidean case, the functional $E_{\text {hyp }}$ is unbounded from below (see Remark A.1). Therefore $U$ does not minimize the energy $E_{\text {hyp }}$ on the Nehari manifold, which in fact fills $\{u \neq$ const. $\}$.

Besides their invariance with respect to Möbius transformations, both system $\left(\mathcal{P}_{0}\right)$ and the related energy $E_{\text {hyp }}$ are invariant with respect to the 3-dimensional group of hyperbolic translations as well. Thus, any $k$-bubble generates a smooth 9-dimensional manifold $Z$ of solutions to $\left(\mathcal{P}_{0}\right)$. We explicitly describe the tangent space $T_{U} Z$ at $U \in Z$ in formula (3.5). 
As a further consequence of the invariances of problem $\left(\mathcal{P}_{0}\right)$, any tangent direction $\varphi \in$ $T_{U} Z$ solves the elliptic system

$$
\Delta \varphi-2 U_{3}^{-1} G^{\prime}(\nabla U) \nabla \varphi=-U_{3}^{-1} \varphi_{3} \Delta U+2 U_{3}^{-1} k\left(\partial_{x} \varphi \wedge \partial_{y} U+\partial_{x} U \wedge \partial_{y} \varphi\right),
$$

which is obtained by linearizing $\left(\mathcal{P}_{0}\right)$ at $U$.

The next one is the main result of the present paper.

Theorem 1.1 (Nondegeneracy) Let $U \in Z$. If $\varphi \in \mathcal{C}^{2}\left(\mathbb{S}^{2}, \mathbb{R}^{3}\right)$ solves the linear system (1.4), then $\varphi \in T_{U} Z$.

Different proofs of the nondegeneracy in the Euclidean case can be found in $[11,15,17]$. The proof of Theorem 1.1 (see Sect. 3), is considerably more involved. It requires the choice of a suitable orthogonal frame for functions in $\mathcal{C}^{2}\left(\mathbb{S}^{2}, \mathbb{R}^{3}\right)$ and the complete classification of solutions of two systems of linear elliptic differential equations, which might have an independent geometrical interest (see Lemmata 3.3, 3.4).

As an application of Theorem 1.1, we provide sufficient conditions on a prescribed smooth function $\phi: \mathbb{H}^{3} \rightarrow \mathbb{R}$ that ensure the existence of embedded surfaces $\mathbb{S}^{2} \rightarrow \mathbb{H}^{3}$ having nonconstant mean curvature $k+\varepsilon \phi$. Our existence results involve the notion of stable critical point already used in [18] and inspired from [2, Chapter 2] (see Sect. 2.2). The main tool is a Lyapunov-Schmidt reduction technique combined with variational arguments, in the spirit of [2].

Theorem 1.2 Let $k>1$ and $\phi \in \mathcal{C}^{1}\left(\mathbb{H}^{3}\right)$ be given. Assume that the function

$$
F_{k}^{\phi}(q):=\int_{\substack{\mathbb{H}\left(\rho_{k} \\(q)\right.}} \phi(p) d \mathbb{H}_{p}^{3}, \quad F_{k}^{\phi}: \mathbb{H}^{3} \rightarrow \mathbb{R}
$$

has a stable critical point in an open set $A \Subset \mathbb{H}^{3}$. For every $\varepsilon \in \mathbb{R}$ close enough to 0 there exist a point $q^{\varepsilon} \in A$, a conformal parametrization $\mathrm{U}_{q^{\varepsilon}}$ of a sphere of radius $\rho_{k}$ about $q^{\varepsilon}$, and a conformally embedded $(k+\varepsilon \phi)$-bubble $u^{\varepsilon}$, such that $\left\|u^{\varepsilon}-\mathrm{U}_{q^{\varepsilon}}\right\|_{\mathcal{C}^{2}}=O(\varepsilon)$ as $\varepsilon \rightarrow 0$.

Moreover, any sequence $\varepsilon_{h} \rightarrow 0$ has a subsequence $\varepsilon_{h_{j}}$ such that $q^{\varepsilon_{h_{j}}}$ converges to a critical point for $F_{k}^{\phi}$. In particular, if $\hat{q} \in A$ is the unique critical point for $F_{k}^{\phi}$ in $\bar{A}$, then $u^{\varepsilon} \rightarrow \mathrm{U}_{\hat{q}}$ in $\mathcal{C}^{2}\left(\mathbb{S}^{2}, \mathbb{H}^{3}\right)$.

Theorem 1.3 Assume that $\phi \in \mathcal{C}^{1}\left(\mathbb{H}^{3}\right)$ has a stable critical point in an open set $A \Subset \mathbb{H}^{3}$. Then there exists $k_{0}>1$ such that for any $k>k_{0}$ and for every $\varepsilon$ close enough to 0 , there exists a conformally embedded $(k+\varepsilon \phi)$-bubble.

In Sect. 4 we first show that the existence of a critical point for $F_{k}^{\phi}(q)$ is a necessary condition in Theorem 1.2. Then we perform the dimension reduction and prove Theorems 1.2, 1.3. With respect to correspondent Euclidean results in [7], a different choice of the functional setting allows us to weaken the regularity assumption on $\phi$ (from $\mathcal{C}^{2}$ to $\mathcal{C}^{1}$ ).

We conclude the paper with an Appendix in which we collect some partially known results about the variational approach to (1.1) and prove a nonexistence result for (1.1) which, in particular, justifies the assumption on the existence of a critical point for $\phi$ in Theorem 1.3.

\section{Notation and preliminaries}

The vector space $\mathbb{R}^{n}$ is endowed with the Euclidean scalar product $\xi \cdot \xi^{\prime}$ and norm $|\xi|$. We denote by $\left\{e_{1}, e_{2}, e_{3}\right\}$ the canonical basis and by $\wedge$ the exterior product in $\mathbb{R}^{3}$. 
We will often identify the complex number $z=x+i y$ with the vector $z=(x, y) \in \mathbb{R}^{2}$. Thus, $i z \equiv(-y, x), z^{2} \equiv\left(x^{2}-y^{2}, 2 x y\right)$ and $z^{-1} \equiv|z|^{-2}(x,-y)$ if $z \neq 0$.

In local coordinates induced by the stereographic projections from the north and the south poles, the round metric on the sphere $\mathbb{S}^{2}$ is given by $g_{h j}=\mu^{2} \delta_{h j}, d \mathbb{S}^{2}=\mu^{2} d z$, where

$$
\mu(z)=\frac{2}{1+|z|^{2}} .
$$

We identify the compactified plane $\overline{\mathbb{R}}^{2}=\mathbb{R}^{2} \cup\{\infty\}$ with the sphere $\mathbb{S}^{2}$ through the inverse of the stereographic projection from the north pole, which is explicitly given by

$$
\omega(x, y)=(\mu x, \mu y, 1-\mu) .
$$

The identity $|\omega|^{2} \equiv 1$ trivially gives $\omega \cdot \partial_{x} \omega \equiv 0, \omega \cdot \partial_{y} \omega \equiv 0$. We also notice that $\omega$ is a conformal (inward-pointing) parametrization of the unit sphere and satisfies

$$
\left\{\begin{array}{l}
\Delta \omega=2 \partial_{x} \omega \wedge \partial_{y} \omega, \quad-\Delta \omega=2 \mu^{2} \omega \\
\partial_{x} \omega \cdot \partial_{y} \omega=0 \\
\left|\partial_{x} \omega\right|^{2}=\left|\partial_{y} \omega\right|^{2}=\frac{1}{2}|\nabla \omega|^{2}=\mu^{2} . \\
\partial_{x} \omega \wedge \omega=\partial_{y} \omega, \quad \omega \wedge \partial_{y} \omega=\partial_{x} \omega, \quad \partial_{x} \omega \wedge \partial_{y} \omega=-\mu^{2} \omega .
\end{array}\right.
$$

\subsection{The Poincaré half-space model}

We adopt as model for the three dimensional hyperbolic space $\mathbb{H}^{3}$ the upper half-space $\mathbb{R}_{+}^{3}=\left\{\left(p_{1}, p_{2}, p_{3}\right) \in \mathbb{R}^{3} \mid p_{3}>0\right\}$ endowed with the Riemannian metric $g_{h j}=p_{3}^{-2} \delta_{h j}$.

The hyperbolic distance $d_{\mathbb{H}}(p, q)$ in $\mathbb{H}^{3}$ is related to the Euclidean one by

$$
\cosh d_{\mathbb{H}}(p, q)=1+\frac{|p-q|^{2}}{2 p_{3} q_{3}},
$$

and the hyperbolic ball $B_{\rho}^{\mathbb{H}}(p)$ centered at $p=\left(p_{1}, p_{2}, p_{3}\right)$ is the Euclidean ball of center $\left(p_{1}, p_{2}, p_{3} \cosh \rho\right)$ and radius $p_{3} \sinh \rho$.

If $F: \mathbb{H}^{3} \rightarrow \mathbb{R}$ is a differentiable function, then $\nabla^{\mathbb{H}} F(p)=p_{3}^{2} \nabla F(p)$, where $\nabla^{\mathbb{H}}, \nabla$ are the hyperbolic and the Euclidean gradients, respectively. In particular, $\nabla^{\mathbb{H}} F(p)=0$ if and only if $\nabla F(p)=0$. The hyperbolic volume form is related to the Euclidean one by $d \mathbb{H}_{p}^{3}=p_{3}^{-3} d p$.

\subsection{Stable critical points}

Let $X \in \mathcal{C}^{1}\left(\mathbb{H}^{3}\right)$ and let $\Omega \Subset \mathbb{H}^{3}$ be open. We say that $X$ has a stable critical point in $\Omega$ if there exists $r>0$ such that any function $G \in \mathcal{C}^{1}(\bar{\Omega})$ satisfying $\|G-X\|_{\mathcal{C}^{1}(\bar{\Omega})}<r$ has a critical point in $\Omega$.

As noticed in [18], conditions to have the existence of a stable critical point $p \in \Omega$ for $X$ are easily given via elementary calculus. For instance, one can use Browder's topological degree theory or can assume that

$$
\min _{\partial \Omega} X>\min _{\Omega} X \text { or } \max _{\partial \Omega} X<\max _{\Omega} X .
$$

Finally, if $X$ is of class $\mathcal{C}^{2}$ and $\Omega$ contains a nondegenerate critical point $p_{0}$ (i.e. the Hessian matrix of $X$ at $p_{0}$ is invertible), then $p_{0}$ is stable. 


\subsection{Function spaces}

Any function $f$ on $\overline{\mathbb{R}}^{2}$ is identified with $f \circ \omega^{-1}$, which is a function on $\mathbb{S}^{2}$. If no confusion can arise, from now on we write $f$ instead of $f \circ \omega^{-1}$.

The Hilbertian norm on $L^{2}\left(\overline{\mathbb{R}}^{2}, \mathbb{R}^{n}\right) \equiv L^{2}\left(\mathbb{S}^{2}, \mathbb{R}^{n}\right)$ is given by

$$
\|f\|_{2}^{2}=\int_{\mathbb{R}^{2}}|f|^{2} \mu^{2} d z<\infty
$$

Let $m \geq 0$. We endow

$$
\mathcal{C}^{m}\left(\overline{\mathbb{R}}^{2}, \mathbb{R}^{n}\right)=\left\{u \in \mathcal{C}^{m}\left(\mathbb{R}^{2}, \mathbb{R}^{n}\right) \mid u\left(z^{-1}\right) \in \mathcal{C}^{m}\left(\mathbb{R}^{2}, \mathbb{R}^{n}\right)\right\} \equiv \mathcal{C}^{m}\left(\mathbb{S}^{2}, \mathbb{R}^{n}\right)
$$

with the standard Banach space structure (we agree that $\mathcal{C}^{m}\left(\overline{\mathbb{R}}^{2}, \mathbb{R}^{n}\right)=\mathcal{C}^{\lfloor m\rfloor, m-\lfloor m\rfloor}\left(\overline{\mathbb{R}}^{2}, \mathbb{R}^{n}\right)$ if $m$ is not an integer). If $m$ is an integer, a norm in $\mathcal{C}^{m}\left(\overline{\mathbb{R}}^{2}, \mathbb{R}^{n}\right)$ is given by

$$
\|u\|_{\mathcal{C}^{m}}=\sum_{j=0}^{m}\left\|\mu^{-j} \nabla^{j} u\right\|_{\infty}
$$

Since we adopt the upper half-space model for $\mathbb{H}^{3}$, we are allowed to write

$$
\mathcal{C}^{m}\left(\overline{\mathbb{R}}^{2}, \mathbb{H}^{3}\right)=\mathcal{C}^{m}\left(\overline{\mathbb{R}}^{2}, \mathbb{R}_{+}^{3}\right)=\left\{u \in \mathcal{C}^{m}\left(\overline{\mathbb{R}}^{2}, \mathbb{R}^{3}\right) \mid u_{3}>0\right\},
$$

so that $\mathcal{C}^{m}\left(\overline{\mathbb{R}}^{2}, \mathbb{H}^{3}\right)$ is an open subset of $\mathcal{C}^{m}\left(\overline{\mathbb{R}}^{2}, \mathbb{R}^{3}\right)$.

If $\psi, \varphi \in \mathcal{C}^{1}\left(\overline{\mathbb{R}}^{2}, \mathbb{R}^{3}\right)$ and $\tau \in \mathbb{R}^{2}$, we put

$$
\nabla \psi \cdot \nabla \varphi=\partial_{x} \psi \cdot \partial_{x} \varphi+\partial_{y} \psi \cdot \partial_{y} \varphi, \quad \tau \nabla \varphi=\tau_{1} \partial_{x} \varphi+\tau_{2} \partial_{y} \varphi
$$

(notice that $\tau \nabla \varphi(z)=d \varphi(z) \tau$ for any $z \in \mathbb{R}^{2}$ ). For instance, we have

$$
z^{h} \nabla \varphi=\left\{\begin{array}{ll}
\partial_{x} \varphi & \text { if } h=0 \\
x \partial_{x} \varphi+y \partial_{y} \varphi & \text { if } h=1
\end{array}, \quad i z^{h} \nabla \varphi= \begin{cases}\partial_{y} \varphi & \text { if } h=0 \\
-y \partial_{x} \varphi+x \partial_{y} \varphi & \text { if } h=1 .\end{cases}\right.
$$

For future convenience we notice, without proof, that the next identities hold:

$$
\left\{\begin{array} { r l } 
{ \partial _ { x } \omega } & { = e _ { 1 } - \omega _ { 1 } \omega - e _ { 2 } \wedge \omega } \\
{ z \nabla \omega } & { = e _ { 3 } - \omega _ { 3 } \omega } \\
{ z ^ { 2 } \nabla \omega } & { = - ( e _ { 1 } - \omega _ { 1 } \omega + e _ { 2 } \wedge \omega ) }
\end{array} \quad \left\{\begin{array}{rl}
\partial_{y} \omega & =e_{2}-\omega_{2} \omega+e_{1} \wedge \omega \\
i z \nabla \omega & =e_{3} \wedge \omega, \\
i z^{2} \nabla \omega & =e_{2}-\omega_{2} \omega-e_{1} \wedge \omega .
\end{array}\right.\right.
$$

The monograph [4] is our reference text for the theory of Sobolev spaces on Riemannian manifolds. In view of our purposes, it is important to notice that

$$
H^{1}\left(\overline{\mathbb{R}}^{2}, \mathbb{R}^{n}\right)=\left\{u \in H_{\mathrm{loc}}^{1}\left(\mathbb{R}^{2}, \mathbb{R}^{n}\right)|| \nabla u|+| u \mid \mu \in L^{2}\left(\mathbb{R}^{2}\right)\right\} \equiv H^{1}\left(\mathbb{S}^{2}, \mathbb{R}^{n}\right) .
$$

We simply write $L^{2}\left(\overline{\mathbb{R}}^{2}\right), \mathcal{C}^{m}\left(\overline{\mathbb{R}}^{2}\right)$ and $H^{1}\left(\overline{\mathbb{R}}^{2}\right)$ instead of $L^{2}\left(\overline{\mathbb{R}}^{2}, \mathbb{R}\right), \mathcal{C}^{m}\left(\overline{\mathbb{R}}^{2}, \mathbb{R}\right)$ and $H^{1}\left(\overline{\mathbb{R}}^{2}, \mathbb{R}\right)$, respectively.

\subsection{Möbius transformations and hyperbolic translations}

Transformations in $P G L(2, \mathbb{C})$ are obtained by composing translations, dilations, rotations and complex inversion. Its Lie algebra admits as a basis the transforms

$$
z \mapsto 1, \quad z \mapsto i, \quad z \mapsto z, \quad z \mapsto i z, \quad z \mapsto z^{2}, \quad z \mapsto i z^{2}
$$


Therefore, for any $u \in \mathcal{C}^{1}\left(\overline{\mathbb{R}}^{2}, \mathbb{H}^{3}\right)$, the functions

$$
z^{h} \nabla u, \quad i z^{h} \nabla u, \quad h=0,1,2,
$$

span the tangent space to the manifold $\{u \circ g \mid g \in P G L(2, \mathbb{C})\}$ at $u$.

Hyperbolic translations are obtained by composing a horizontal (Euclidean) translation $p \mapsto p+a e_{1}+b e_{2}, a, b \in \mathbb{R}$ with an Euclidean homothety $p \mapsto t p, t>0$. Therefore, for any $u \in \mathcal{C}^{m}\left(\overline{\mathbb{R}}^{2}, \mathbb{H}^{3}\right)$, the functions

$$
e_{1}, \quad e_{2}, u,
$$

span the tangent space to the manifold $\left\{u_{q} \mid q \in \mathbb{H}^{3}\right\}$ at $u$, where

$$
u_{q}:=q_{3} u+q-\left(q \cdot e_{3}\right) e_{3} \text {. }
$$

\section{Nondegeneracy of hyperbolic $\boldsymbol{k}$-bubbles}

The proof of Theorem 1.1 needs some preliminary work. We put

$$
\mathrm{U}=r_{k}\left(\omega+k e_{3}\right), \quad r_{k}:=\sinh \rho_{k}=\frac{1}{k} \cosh \rho_{k}=\frac{1}{\sqrt{k^{2}-1}},
$$

where $\omega$ is given by (2.1). Since $U$ is a conformal parametrization of the Euclidean sphere of radius $r_{k}$ about $k r_{k} e_{3}$, which coincides with the hyperbolic sphere of radius $\rho_{k}$ about $e_{3}$, then $\mathrm{U}$ has curvature $k$ and in fact it solves $\left(\mathcal{P}_{0}\right)$. Accordingly with (2.5), we put

$$
\mathrm{U}_{q}:=q_{3} \mathrm{U}+q-\left(q \cdot e_{3}\right) e_{3}
$$

(notice that $\mathrm{U}_{e_{3}}=\mathrm{U}$ ), and introduce the 9-dimensional manifold

$$
Z=\left\{\mathrm{U}_{q} \circ g \mid g \in P G L(2, \mathbb{C}), q \in \mathbb{H}^{3}\right\} .
$$

Remark 3.1 Any surface $U \in Z$ is an embedding and solves $\left(\mathcal{P}_{0}\right)$. Conversely, let $U \in$ $\mathcal{C}^{2}\left(\overline{\mathbb{R}}^{2}, \mathbb{H}^{3}\right)$ be an embedding. If $U$ solves $\left(\mathcal{P}_{0}\right)$, then it is a $k$-bubble by Lemma A.2 and, thanks to an Alexandrov' type argument (see for instance [16, Corollary 10.3.2]) it parametrizes a sphere of hyperbolic radius $\rho_{k}$ and Euclidean radius $r_{k}$. Since $U$ is conformal, then $\Delta U=2 r_{k}^{-1} \partial_{x} U \wedge \partial_{y} U$. Therefore $U \in Z$ by the uniqueness result in [5].

By the remarks in Sect. 2.4 and since $\nabla \mathrm{U}_{q}$ is proportional to $\nabla \omega$, we have that $T_{\mathrm{U}_{q}} Z=$ $T_{\mathrm{U}} Z$ for any $q \in \mathbb{H}^{3}$, and

$$
T_{\mathrm{U}} Z=\left\langle\left\{z^{h} \nabla \omega, i z^{h} \nabla \omega \mid h=0,1,2\right\}\right\rangle \oplus\left\langle e_{1}, e_{2}, \mathrm{U}\right\rangle .
$$

Moreover, any tangent direction $\tau \in T_{\mathrm{U}} Z$ solves (1.4).

It is convenient to split $\mathcal{C}^{m}\left(\overline{\mathbb{R}}^{2}, \mathbb{R}^{3}\right)$ in the direct sum of its closed subspaces

$$
\langle\omega\rangle_{\mathcal{C}^{m}}:=\left\{\varphi \in \mathcal{C}^{m}\left(\overline{\mathbb{R}}^{2}, \mathbb{R}^{3}\right) \mid \varphi \cdot \omega \equiv 0 \text { on } \mathbb{R}^{2}\right\}, \quad\langle\omega\rangle_{\mathcal{C}^{m}}:=\left\{\eta \omega \mid \eta \in \mathcal{C}^{m}\left(\overline{\mathbb{R}}^{2}\right)\right\} .
$$

Since $T_{\mathrm{U}} Z=\left(T_{\mathrm{U}} Z \cap\langle\omega\rangle_{C^{2}}^{\perp}\right) \oplus\left(T_{\mathrm{U}} Z \cap\langle\omega\rangle_{C^{2}}\right)$, from (2.4) we infer another useful description of the tangent space, that is

$$
T_{\mathrm{U}} Z=\left\{s-(s \cdot \omega) \omega+t \wedge \omega \mid s, t \in \mathbb{R}^{3}\right\} \oplus\left\{\left(\alpha \cdot\left(k \omega+e_{3}\right)\right) \omega \mid \alpha \in \mathbb{R}^{3}\right\} .
$$

We now introduce the differential operator

$$
J_{0}(u)=-\operatorname{div}\left(u_{3}^{-2} \nabla u\right)-u_{3}^{-3}|\nabla u|^{2} e_{3}+2 k u_{3}^{-3} \partial_{x} u \wedge \partial_{y} u .
$$


Notice that $Z \subset\left\{J_{0}=0\right\}$. Further, let $I(z)=z^{-1}$. Since $J_{0}(u \circ I)=|z|^{-4} J_{0}(u) \circ I$ for any $u \in \mathcal{C}^{2+m}\left(\overline{\mathbb{R}}^{2}, \mathbb{H}^{3}\right), m \geq 0$, then $J_{0}$ is a $\mathcal{C}^{1}$ map

$$
J_{0}: \mathcal{C}^{2+m}\left(\overline{\mathbb{R}}^{2}, \mathbb{H}^{3}\right) \rightarrow \mathcal{C}^{m}\left(\overline{\mathbb{R}}^{2}, \mathbb{R}^{3}\right) .
$$

We denote by $J_{0}^{\prime}(u): \mathcal{C}^{2+m}\left(\overline{\mathbb{R}}^{2}, \mathbb{R}^{3}\right) \rightarrow \mathcal{C}^{m}\left(\overline{\mathbb{R}}^{2}, \mathbb{R}^{3}\right)$ its differential at $u$.

Finally, $J_{0}\left(\mathrm{U}_{q} \circ g\right)=0$ for any $g \in P G L(2, \mathbb{C}), q \in \mathbb{H}^{3}$, that implies $T_{\mathrm{U}} Z \subseteq \operatorname{ker} J_{0}^{\prime}(\mathrm{U})$. In order to prove Theorem 1.1 it suffices to show that

$$
\operatorname{ker} J_{0}^{\prime}(\mathrm{U}) \subseteq T_{\mathrm{U}} Z
$$

\section{Main computations}

Recall that $\mathrm{U}=r_{k}\left(\omega+k e_{3}\right)$ solves $J_{0}(\mathrm{U})=0$ to check that

$$
\begin{aligned}
J_{0}^{\prime}(\mathrm{U}) \varphi= & -\operatorname{div}\left(\mathrm{U}_{3}^{-2} \nabla \varphi\right) \\
& +2 \mathrm{U}_{3}^{-3}\left[G^{\prime}(\nabla \mathrm{U}) \nabla \varphi-\nabla U_{3} \nabla \varphi-\frac{1}{2} \varphi_{3} \Delta \mathrm{U}+k\left(\partial_{x} \varphi \wedge \partial_{y} \mathrm{U}+\partial_{x} \mathrm{U} \wedge \partial_{y} \varphi\right)\right],
\end{aligned}
$$

where $G$ is given by (1.2). Since $\nabla \omega_{3}=-\nabla \mu=\mu^{2} z$, thanks to (2.2) we have

$$
\begin{aligned}
& r_{k}^{2} J_{0}^{\prime}(\mathrm{U}) \varphi=-\operatorname{div}\left(\left(\omega_{3}+k\right)^{-2} \nabla \varphi\right) \\
& \quad+2\left(\omega_{3}+k\right)^{-3}\left[\left(G^{\prime}(\nabla \omega) \nabla \varphi-\mu^{2} z \nabla \varphi\right)+\mu^{2} \varphi_{3} \omega+k\left(\partial_{x} \varphi \wedge \partial_{y} \omega+\partial_{x} \omega \wedge \partial_{y} \varphi\right)\right] \\
& G^{\prime}(\nabla \omega) \nabla \varphi-\mu^{2} z \nabla \varphi=\nabla \varphi_{3} \nabla \omega-(\nabla \varphi \cdot \nabla \omega) e_{3} .
\end{aligned}
$$

To rewrite (3.6) in a less obscure form, we decompose any $\varphi \in \mathcal{C}^{m}\left(\overline{\mathbb{R}}^{2}, \mathbb{R}^{3}\right), m \geq 0$, as

$$
\varphi=\mathcal{P} \varphi+(\varphi \cdot \omega) \omega, \quad \mathcal{P} \varphi:=\varphi-(\varphi \cdot \omega) \omega=\mu^{-2}\left(\left(\varphi \cdot \partial_{x} \omega\right) \partial_{x} \omega+\left(\varphi \cdot \partial_{y} \omega\right) \partial_{y} \omega\right),
$$

compare with (3.4). Accordingly, for $\varphi \in \mathcal{C}^{2}\left(\overline{\mathbb{R}}^{2}, \mathbb{R}^{3}\right)$ we have

$$
J_{0}^{\prime}(\mathrm{U}) \varphi=\mathcal{P}\left(J_{0}^{\prime}(\mathrm{U}) \varphi\right)+\left(J_{0}^{\prime}(\mathrm{U}) \varphi \cdot \omega\right) \omega
$$

so that we can reconstruct $J_{0}^{\prime}(\mathrm{U}) \varphi \in \mathcal{C}^{0}\left(\overline{\mathbb{R}}^{2}, \mathbb{R}^{3}\right)$ by providing explicit expressions for $\mathcal{P}\left(J_{0}^{\prime}(\mathrm{U}) \varphi\right)$ and $J_{0}^{\prime}(\mathrm{U}) \varphi \cdot \omega$, separately. This will be done in the next Lemma.

Lemma 3.1 Let $\varphi \in \mathcal{C}^{2}\left(\overline{\mathbb{R}}^{2}, \mathbb{R}^{3}\right)$. Then

$$
\begin{aligned}
& r_{k}^{2} \mathcal{P}\left(J_{0}^{\prime}(\mathrm{U}) \varphi\right)=\mathcal{P}\left(-\operatorname{div}\left(\frac{\nabla \mathcal{P} \varphi}{\left(\omega_{3}+k\right)^{2}}\right)\right)+\frac{2 \mu^{2}}{\left(\omega_{3}+k\right)^{3}}(i z \nabla \mathcal{P} \varphi) \wedge \omega-\frac{2 \mu^{2}}{\left(\omega_{3}+k\right)^{2}} \mathcal{P} \varphi, \\
& r_{k}^{2}\left(J_{0}^{\prime}(\mathrm{U}) \varphi\right) \cdot \omega=-\operatorname{div}\left(\frac{\nabla(\varphi \cdot \omega)}{\left(\omega_{3}+k\right)^{2}}\right)-\frac{2 k \mu^{2}}{\left(\omega_{3}+k\right)^{3}}(\varphi \cdot \omega) .
\end{aligned}
$$

Proof We introduce the differential operator $L=-\operatorname{div}\left(\left(\omega_{3}+k\right)^{-2} \nabla\right)$ and start to prove (3.10) by noticing that

$$
L \varphi \cdot \omega=L(\varphi \cdot \omega)+2\left(\omega_{3}+k\right)^{-3}\left[\left(\omega_{3}+k\right) \nabla \varphi \cdot \nabla \omega-\mu^{2} \varphi \cdot(z \nabla \omega)-\mu^{2}\left(\omega_{3}+k\right)(\varphi \cdot \omega)\right] .
$$

Recalling that $\omega$ is pointwise orthogonal to $\partial_{x} \omega, \partial_{y} \omega$, from (3.7) we obtain

$$
\left(G^{\prime}(\nabla \omega) \nabla \varphi-\mu^{2} z \nabla \varphi\right) \cdot \omega=-(\nabla \varphi \cdot \nabla \omega) \omega_{3} .
$$


Further, by (2.2) we have $\left(\partial_{x} \varphi \wedge \partial_{y} \omega+\partial_{x} \omega \wedge \partial_{y} \varphi\right) \cdot \omega=-\nabla \varphi \cdot \nabla \omega$. Finally, we obtain

$$
r_{k}^{2}\left(J_{0}^{\prime}(\mathrm{U}) \varphi\right) \cdot \omega=L(\varphi \cdot \omega)-2\left(\omega_{3}+k\right)^{-3} \mu^{2}\left[\varphi \cdot(z \nabla \omega)-\varphi_{3}+\left(\omega_{3}+k\right)(\varphi \cdot \omega)\right],
$$

and (3.10) follows, because $e_{3}=z \nabla \omega+\omega_{3} \omega$, see (2.4).

Next, using the equivalent formulation

$$
\mathrm{U}_{3}^{2} J_{0}^{\prime}(\mathrm{U}) \varphi=-\Delta \varphi+2\left(\omega_{3}+k\right)^{-1}\left[G^{\prime}(\nabla \omega) \nabla \varphi+\mu^{2} \omega \varphi_{3}+k\left(\partial_{x} \varphi \wedge \partial_{y} \omega+\partial_{x} \omega \wedge \partial_{y} \varphi\right)\right]
$$

we find that, for $\varphi=\eta \omega, \eta \in \mathcal{C}^{2}\left(\overline{\mathbb{R}}^{2}\right)$, it holds

$$
\mathrm{U}_{3}^{2} J_{0}^{\prime}(\mathrm{U})(\eta \omega) \cdot \partial_{x} \omega=\mathrm{U}_{3}^{2} J_{0}^{\prime}(\mathrm{U})(\eta \omega) \cdot \partial_{y} \omega=0,
$$

whence we infer

$$
\mathcal{P}\left(J_{0}^{\prime}(\mathrm{U})(\varphi-\mathcal{P} \varphi)\right)=0, \quad \text { for every } \varphi \in \mathcal{C}^{2}\left(\overline{\mathbb{R}}^{2}, \mathbb{R}^{3}\right) .
$$

Thanks to (3.10) and (3.12) we get $\mathcal{P}\left(J_{0}^{\prime}(\mathrm{U}) \varphi\right)=J_{0}^{\prime}(\mathrm{U})(\mathcal{P} \varphi)$, thus to conclude the proof we can assume that $\varphi=\mathcal{P} \varphi$. Since $\varphi$ is pointwise orthogonal to $\omega$, we trivially have

$$
\partial_{x} \varphi \cdot \omega=-\varphi \cdot \partial_{x} \omega, \quad \partial_{y} \varphi \cdot \omega=-\varphi \cdot \partial_{y} \omega .
$$

We start to handle (3.7). From $e_{3}=z \nabla \omega+\omega_{3} \omega$ we get

$$
\begin{aligned}
& \left(G^{\prime}(\nabla \omega) \nabla \varphi-\mu^{2} z \nabla \varphi\right)+\omega_{3}(\nabla \varphi \cdot \nabla \omega) \omega=\nabla \varphi_{3} \nabla \omega-(\nabla \varphi \cdot \nabla \omega) z \nabla \omega \\
& =\left(\partial_{x} \varphi_{3}-x(\nabla \varphi \cdot \nabla \omega)\right) \partial_{x} \omega+\left(\partial_{y} \varphi_{3}-y(\nabla \varphi \cdot \nabla \omega)\right) \partial_{y} \omega .
\end{aligned}
$$

Further,

$$
\begin{aligned}
& \partial_{x} \varphi_{3}-x(\nabla \varphi \cdot \nabla \omega)=\partial_{x} \varphi \cdot\left(z \nabla \omega+\omega_{3} \omega\right)-x(\nabla \varphi \cdot \nabla \omega) \\
& \quad=\left(\partial_{x} \varphi \cdot(z \nabla \omega)-x(\nabla \varphi \cdot \nabla \omega)\right)-\omega_{3} \varphi \cdot \partial_{x} \omega=-(i z \nabla \varphi) \cdot \partial_{y} \omega-\omega_{3} \varphi \cdot \partial_{x} \omega .
\end{aligned}
$$

In a similar way one can check that $\partial_{y} \varphi_{3}-y(\nabla \varphi \cdot \nabla \omega)=(i z \nabla \varphi) \cdot \partial_{x} \omega-\omega_{3} \varphi \cdot \partial_{y} \omega$, thus

$$
G^{\prime}(\nabla \omega) \nabla \varphi-\mu^{2} z \nabla \varphi=\mu^{2}(i z \nabla \varphi) \wedge \omega-\omega_{3}(\nabla \varphi \cdot \nabla \omega) \omega-\mu^{2} \omega_{3} \varphi
$$

Next, using (2.2) we can compute

$$
\begin{aligned}
& \partial_{x} \varphi \wedge \partial_{y} \omega=\partial_{x} \varphi \wedge\left(\partial_{x} \omega \wedge \omega\right)=-\left(\varphi \cdot \partial_{x} \omega\right) \partial_{x} \omega-\left(\partial_{x} \varphi \cdot \partial_{x} \omega\right) \omega \\
& \partial_{x} \omega \wedge \partial_{y} \varphi=\left(\omega \wedge \partial_{y} \omega\right) \wedge \partial_{y} \varphi=-\left(\varphi \cdot \partial_{y} \omega\right) \partial_{y} \omega-\left(\partial_{y} \varphi \cdot \partial_{y} \omega\right) \omega
\end{aligned}
$$

which give the identity

$$
\partial_{x} \varphi \wedge \partial_{y} \omega+\partial_{x} \omega \wedge \partial_{y} \varphi=-\mu^{2} \varphi-(\nabla \varphi \cdot \nabla \omega) \omega,
$$

that holds for any $\varphi \in\langle\omega\rangle_{\mathcal{C}^{m}}$.

Putting together the above informations we arrive at

$$
\begin{aligned}
r_{k}^{2} J_{0}^{\prime}(\mathrm{U}) \varphi= & L \varphi+\frac{2 \mu^{2}}{\left(\omega_{3}+k\right)^{3}}(i z \nabla \varphi) \wedge \omega-\frac{2 \mu^{2}}{\left(\omega_{3}+k\right)^{2}} \varphi \\
& +\frac{2}{\left(\omega_{3}+k\right)^{3}}\left[\mu^{2} \varphi_{3}-\left(\omega_{3}+k\right) \nabla \varphi \cdot \nabla \omega\right] \omega .
\end{aligned}
$$

Using (3.11) and $\varphi_{3}=\varphi \cdot(z \nabla \omega)$, we conclude the proof. 
Thanks to Lemma 3.1 we can study the system $J_{0}^{\prime}(\mathrm{U}) \varphi=0$ separately, on $\langle\omega\rangle \frac{\perp}{\mathcal{C}^{m}}$ first, and on $\langle\omega\rangle_{\mathcal{C}^{m}}$ later. In fact, $\varphi \in \operatorname{ker} J_{0}^{\prime}(\mathrm{U})$ if and only if the pair of functions

$$
\psi:=\mathcal{P} \varphi \in\langle\omega\rangle_{\mathcal{C}^{2}}^{\perp} \subset \mathcal{C}^{2}\left(\overline{\mathbb{R}}^{2}, \mathbb{R}^{3}\right), \quad \eta:=\varphi \cdot \omega \in \mathcal{C}^{2}\left(\overline{\mathbb{R}}^{2}\right),
$$

solves

$$
\left\{\begin{array}{l}
\mathcal{P}\left(-\operatorname{div}\left(\frac{\nabla \psi}{\left(\omega_{3}+k\right)^{2}}\right)\right)+\frac{2 \mu^{2}}{\left(\omega_{3}+k\right)^{3}}(i z \nabla \psi) \wedge \omega=\frac{2 \mu^{2}}{\left(\omega_{3}+k\right)^{2}} \psi \\
-\operatorname{div}\left(\frac{\nabla \eta}{\left(\omega_{3}+k\right)^{2}}\right)=\frac{2 k \mu^{2}}{\left(\omega_{3}+k\right)^{3}} \eta
\end{array}\right.
$$

We begin by facing problem (3.14a). Firstly, we show that the quadratic form associated to the differential operator $J_{0}^{\prime}(\mathrm{U})$ is nonnegative on $\langle\omega\rangle \frac{\perp}{\mathcal{C}^{2}}$.

Lemma 3.2 Let $\psi \in\langle\omega\rangle \frac{\perp}{\mathcal{C}^{2}}$. Then

$$
\int_{\mathbb{R}^{2}} J_{0}^{\prime}(\mathrm{U}) \psi \cdot \psi d z=r_{k}^{-2} \int_{\mathbb{R}^{2}} \frac{\left(\partial_{x} \psi \cdot \partial_{x} \omega-\partial_{y} \psi \cdot \partial_{y} \omega\right)^{2}+\left(\partial_{x} \psi \cdot \partial_{y} \omega+\partial_{y} \psi \cdot \partial_{x} \omega\right)^{2}}{\mu^{2}\left(\omega_{3}+k\right)^{2}} d z .
$$

Proof Since $J_{0}^{\prime}(\mathrm{U}) \psi \cdot \psi=\mathcal{P}\left(J_{0}^{\prime}(\mathrm{U}) \psi\right) \cdot \psi$ and $\mathcal{P} \psi=\psi$, formula (3.9) gives

$$
r_{k}^{2} \int_{\mathbb{R}^{2}} J_{0}^{\prime}(\mathrm{U}) \psi \cdot \psi d z=\int_{\mathbb{R}^{2}} \frac{|\nabla \psi|^{2}}{\left(\omega_{3}+k\right)^{2}} d z+2 \int_{\mathbb{R}^{2}} \frac{\psi \cdot(i z \nabla \psi) \wedge \omega}{\left(\omega_{3}+k\right)^{3}} \mu^{2} d z-2 \int_{\mathbb{R}^{2}} \frac{|\psi|^{2}}{\left(\omega_{3}+k\right)^{2}} \mu^{2} d z .
$$

Now we prove the identity

$$
B_{\psi}:=2 \int_{\mathbb{R}^{2}} \frac{\psi \cdot(i z \nabla \psi) \wedge \omega}{\left(\omega_{3}+k\right)^{3}} \mu^{2} d z=2 \int_{\mathbb{R}^{2}} \frac{\omega \cdot \partial_{x} \psi \wedge \partial_{y} \psi}{\left(\omega_{3}+k\right)^{2}} d z+\int_{\mathbb{R}^{2}} \frac{|\psi|^{2}}{\left(\omega_{3}+k\right)^{2}} \mu^{2} d z .
$$

We use polar coordinates $\rho, \theta$ on $\mathbb{R}^{2}$ and notice that $\partial_{\theta} \psi=i z \nabla \psi$. From $\rho \mu^{2}=\partial_{\rho} \omega_{3}$ we get

$$
\begin{aligned}
B_{\psi}= & -\int_{0}^{2 \pi} d \theta \int_{0}^{\infty}\left(\psi \cdot \partial_{\theta} \psi \wedge \omega\right) \partial_{\rho}\left(\omega_{3}+k\right)^{-2} d \rho \\
= & \int_{0}^{\infty} d \rho \int_{0}^{2 \pi} \frac{\omega \cdot \partial_{\rho} \psi \wedge \partial_{\theta} \psi-\psi \cdot \partial_{\rho} \omega \wedge \partial_{\theta} \psi}{\left(\omega_{3}+k\right)^{2}} d \theta+\int_{0}^{\infty} d \rho \int_{0}^{2 \pi} \frac{\partial_{\rho \theta} \psi \cdot \omega \wedge \psi}{\left(\omega_{3}+k\right)^{2}} d \theta \\
= & \int_{0}^{\infty} d \rho \int_{0}^{2 \pi} \frac{\omega \cdot \partial_{\rho} \psi \wedge \partial_{\theta} \psi-\psi \cdot \partial_{\rho} \omega \wedge \partial_{\theta} \psi}{\left(\omega_{3}+k\right)^{2}} d \theta \\
& \quad+\int_{0}^{\infty} d \rho \int_{0}^{2 \pi} \frac{\omega \cdot \partial_{\rho} \psi \wedge \partial_{\theta} \psi-\psi \cdot \partial_{\rho} \psi \wedge \partial_{\theta} \omega}{\left(\omega_{3}+k\right)^{2}} d \theta .
\end{aligned}
$$

Using the elementary identity $\partial_{\rho} \alpha \wedge \partial_{\theta} \beta=\rho\left(\partial_{x} \alpha \wedge \partial_{y} \beta\right)$, we see that

$$
B_{\psi}=2 \int_{\mathbb{R}^{2}} \frac{\omega \cdot \partial_{x} \psi \wedge \partial_{y} \psi}{\left(\omega_{3}+k\right)^{2}} d z-\int_{\mathbb{R}^{2}} \frac{\psi \cdot\left(\partial_{x} \omega \wedge \partial_{y} \psi+\partial_{x} \psi \wedge \partial_{y} \omega\right)}{\left(\omega_{3}+k\right)^{2}} d z
$$


and (3.15) follows from (3.13) (with $\varphi$ replaced by $\psi$ ).

Thanks to (3.15), we have the identity

$$
r_{k}^{2} \int_{\mathbb{R}^{2}} J_{0}^{\prime}(\mathrm{U}) \psi \cdot \psi d z=\int_{\mathbb{R}^{2}} \frac{|\nabla \psi|^{2}+2 \omega \cdot \partial_{x} \psi \wedge \partial_{y} \psi-\mu^{2}|\psi|^{2}}{\left(\omega_{3}+k\right)^{2}} d z,
$$

so that we only need to handle the function

$$
b_{\psi}:=|\nabla \psi|^{2}+2 \omega \cdot \partial_{x} \psi \wedge \partial_{y} \psi-\mu^{2}|\psi|^{2} .
$$

We decompose $\partial_{x} \psi$ and $\partial_{y} \psi$ accordingly with (3.8), to obtain

$$
\begin{aligned}
& \mu^{2} \partial_{x} \psi=\left(\partial_{x} \psi \cdot \partial_{x} \omega\right) \omega_{x}+\left(\partial_{x} \psi \cdot \partial_{y} \omega\right) \omega_{y}-\mu^{2}\left(\psi \cdot \partial_{x} \omega\right) \omega, \\
& \mu^{2} \partial_{y} \psi=\left(\partial_{y} \psi \cdot \partial_{x} \omega\right) \omega_{x}+\left(\partial_{y} \psi \cdot \partial_{y} \omega\right) \omega_{y}-\mu^{2}\left(\psi \cdot \partial_{y} \omega\right) \omega,
\end{aligned}
$$

respectively. Since $|\nabla \psi|^{2}=\left|\partial_{x} \psi\right|^{2}+\left|\partial_{y} \psi\right|^{2}$, we infer

$$
\mu^{2}\left(|\nabla \psi|^{2}-\mu^{2}|\psi|^{2}\right)=\left(\partial_{x} \psi \cdot \partial_{x} \omega\right)^{2}+\left(\partial_{x} \psi \cdot \partial_{y} \omega\right)^{2}+\left(\partial_{y} \psi \cdot \partial_{x} \omega\right)^{2}+\left(\partial_{y} \psi \cdot \partial_{y} \omega\right)^{2} .
$$

Writing $\mu^{2} \omega=-\partial_{x} \omega \wedge \partial_{y} \omega$, see (2.2), we get

$$
\mu^{2} \omega \cdot\left(\partial_{x} \psi \wedge \partial_{y} \psi\right)=-\left(\partial_{x} \psi \cdot \partial_{x} \omega\right)\left(\partial_{y} \psi \cdot \partial_{y} \omega\right)+\left(\partial_{x} \psi \cdot \partial_{y} \omega\right)\left(\partial_{y} \psi \cdot \partial_{x} \omega\right)
$$

from which it readily follows that $\mu^{2} b_{\psi}=\left(\partial_{x} \psi \cdot \partial_{x} \omega-\partial_{y} \psi \cdot \partial_{y} \omega\right)^{2}+\left(\partial_{x} \psi \cdot \partial_{y} \omega+\partial_{y} \psi \cdot \partial_{x} \omega\right)^{2}$. The proof is complete.

Lemma 3.3 Let $\psi \in \mathcal{C}^{2}\left(\overline{\mathbb{R}}^{2}, \mathbb{R}^{3}\right)$ be a solution to $(3.14 \mathrm{a})$. There exist $s, t \in \mathbb{R}^{3}$ such that

$$
\psi=s-(s \cdot \omega) \omega+t \wedge \omega,
$$

and thus $\psi \in T_{\mathrm{U}} Z \cap\langle\omega\rangle \underset{C^{2}}{\perp}=\left\{s-(s \cdot \omega) \omega+t \wedge \omega \mid s, t \in \mathbb{R}^{3}\right\}$.

Proof From (3.14a) it immediately follows that $\psi$ is pointwise orthogonal to $\omega$, which implies $\psi \in\langle\omega\rangle_{\mathcal{C}^{2}}^{\perp}$. Since $\mathcal{P} \psi=\psi$, then $J_{0}^{\prime}(\mathrm{U}) \psi=0$ by (3.9) and (3.10), hence

$$
\left\{\begin{array}{l}
\partial_{x} \psi \cdot \partial_{x} \omega-\partial_{y} \psi \cdot \partial_{y} \omega=0 \\
\partial_{x} \psi \cdot \partial_{y} \omega+\partial_{y} \psi \cdot \partial_{x} \omega=0
\end{array}\right.
$$

by Lemma 3.2. Since $\psi \in\left\langle\partial_{x} \omega, \partial_{y} \omega\right\rangle$ pointwise on $\mathbb{R}^{2}$, we can write

$$
\psi=f \nabla \omega, \text { where } f:=\mu^{-2}\left(\psi \cdot \partial_{x} \omega, \psi \cdot \partial_{y} \omega\right) \in \mathcal{C}^{2}\left(\overline{\mathbb{R}}^{2}, \mathbb{R}^{2}\right) .
$$

We identify $f$ with a complex valued function. A direct computation based on (2.2) shows that $\psi$ solves (3.16) if and only if $f$ solves $\partial_{x} f+i \partial_{y} f=0$ on $\mathbb{R}^{2}$. In polar coordinates we have that

$$
\rho \partial_{\rho} f+i \partial_{\theta} f=0 .
$$

For every $\rho>0$ we expand the periodic function $f(\rho, \cdot)$ in Fourier series,

$$
f(\rho, \theta)=\sum_{h \in \mathbb{Z}} \gamma_{h}(\rho) e^{i h \theta}, \quad \gamma_{h}(\rho)=\frac{1}{2 \pi} \int_{0}^{2 \pi} f(\rho, \theta) e^{-i h \theta} d \theta .
$$

The coefficients $\gamma_{h}$ are complex-valued functions on the half-line $\mathbb{R}_{+}$and solve

$$
\gamma_{h}^{\prime}-h \gamma_{h}=0
$$


because of (3.17). Thus for every $h \in \mathbb{Z}$ there exists $a_{h} \in \mathbb{C}$ such that $\gamma_{h}(\rho)=a_{h} \rho^{h}$. Now recall that $\mu \psi \in L^{2}\left(\mathbb{R}^{2}, \mathbb{R}^{3}\right)$. Since

$$
\int_{\mathbb{R}^{2}} \mu^{2}|\psi|^{2} d z=\int_{\mathbb{R}^{2}} \mu^{4}|f|^{2} d z \geq 2 \pi \int_{0}^{\infty} \mu^{4} \rho\left|\gamma_{h}\right|^{2} d \rho=a_{h}^{2} \int_{\mathbb{R}^{2}} \mu^{4}|z|^{2 h} d z, \quad \forall h \in \mathbb{Z},
$$

we infer that $\gamma_{h}=0$ for every $h \neq 0,1,2$. Thus $f(z)=\sum_{h=0}^{2} a_{h} z^{h}$, that is $\psi=\sum_{h=0}^{2} a_{h} z^{h} \nabla \omega$, and in particular the space of solutions of (3.14a) has (real) dimension 6. The conclusion of the proof follows from the relations (2.4).

Lemma 3.4 Let $\eta \in \mathcal{C}^{2}\left(\overline{\mathbb{R}}^{2}\right)$ be a solution to (3.14b). There exists $\alpha \in \mathbb{R}^{3}$ such that

$$
\eta=\alpha \cdot\left(k \omega+e_{3}\right)
$$

and thus $\eta \omega \in T_{\mathrm{U}} Z \cap\langle\omega\rangle_{\mathcal{C}^{2}}=\left\{\left(\alpha \cdot\left(k \omega+e_{3}\right)\right) \omega \mid \alpha \in \mathbb{R}^{3}\right\}$.

Proof First of all, we notice that $\alpha \cdot\left(k \omega+e_{3}\right)$ solves (3.14b) for any $\alpha \in \mathbb{R}^{3}$.

By the Hilbert-Schmidt theorem, the eigenvalue problem

$$
-\operatorname{div}\left(\frac{\nabla \eta}{\left(\omega_{3}+k\right)^{2}}\right)=\frac{\lambda \mu^{2}}{\left(\omega_{3}+k\right)^{3}} \eta \quad \text { on } \mathbb{R}^{2}, \quad \eta \in \mathcal{C}^{2}\left(\mathbb{R}^{2}\right),
$$

has a non decreasing, divergent sequence $\left(\lambda_{h}\right)_{h \geq 0}$ of eigenvalues which correspond to critical levels of the quotient

$$
R(\eta):=\frac{\int_{\mathbb{R}^{2}} \frac{|\nabla \eta|^{2}}{\left(\omega_{3}+k\right)^{2}} d z}{\int_{\mathbb{R}^{2}} \frac{|\eta|^{2}}{\left(\omega_{3}+k\right)^{3}} \mu^{2} d z}, \quad \eta \in H^{1}\left(\overline{\mathbb{R}}^{2}\right) \backslash\{0\} .
$$

Clearly, $\lambda_{0}=0$ is simple, and its eigenfunctions are constant functions. We claim that the next eigenvalue is $2 k$, and that its eigenspace has dimension 3 , which concludes the proof.

To this goal, we use the functional change

$$
\eta(z)=\frac{\mu(z)}{\mu\left(c_{k} z\right)} \Phi\left(c_{k} z\right), \quad c_{k}:=e^{\rho_{k}}=\sqrt{\frac{k+1}{k-1}} .
$$

By a direct computation involving the identity $\left(\omega_{3}(z)+k\right) \mu\left(c_{k} z\right)=(k-1) \mu(z)$ and integration by parts, one gets

$$
\lambda_{1}=\inf _{\substack{\eta \in \mathcal{C}^{2}\left(\overline{\mathbb{R}}^{2}\right) \backslash\{0\} \\ \int_{\mathbb{R}^{2}} \frac{\eta \mu^{2} d z}{\left(\omega_{3}+k\right)^{3}}=0}} R(\eta)=2 k+\inf _{\substack{\Phi \in \mathcal{C}^{2}\left(\overline{\mathbb{R}}^{2}\right) \backslash\{0\} \\ \int_{\mathbb{R}^{2}} \Phi \mu^{2} d z=0}} \frac{\int_{\mathbb{R}^{2}}|\nabla \Phi|^{2} d z-2 \int_{\mathbb{R}^{2}}|\Phi|^{2} \mu^{2} d z}{\int_{\mathbb{R}^{2}} \frac{|\Phi|^{2}}{\left(k-\omega_{3}\right)} \mu^{2} d z} .
$$

On the other hand, it is well known that

$$
\min _{\substack{\Phi \in \mathcal{C}^{2}\left(\overline{\mathbb{R}}^{2}\right) \backslash\{0\} \\ \int_{\mathbb{R}^{2}} \Phi \mu^{2} d z=0}} \frac{\int_{\mathbb{R}^{2}}|\nabla \Phi|^{2} d z}{\int_{\mathbb{R}^{2}}|\Phi|^{2} \mu^{2} d z}=2
$$

is the first nontrivial eigenvalue for the Laplace-Beltrami operator on the sphere and that its eigenspace has dimension 3, see for instance [4]. The proof is complete. 
Remark 3.2 The third eigenvalue $\lambda_{2}$ of (3.18) verifies $\lambda_{2}>2 k$ by Lemma 3.4, and

$$
\lambda_{2}=\min \left\{R(\eta) \mid \int_{\mathbb{R}^{2}} \frac{\eta}{\left(\omega_{3}+k\right)^{3}} \mu^{2} d z=\int_{\mathbb{R}^{2}} \frac{\eta\left(k \omega_{j}+\delta_{j 3}\right)}{\left(\omega_{3}+k\right)^{3}} \mu^{2} d z=0, \quad j=1,2,3\right\} .
$$

Proof of Theorem 1.1 In fact, we only have to sum up the argument. Let $U \in Z$. Thanks to (3.2), $U=\mathrm{U}_{q} \circ g$ for some $q \in \mathbb{H}^{3}, g \in P G L(2, \mathbb{C})$. Since

$T_{\mathrm{U}_{q} \circ g} Z=T_{\mathrm{U}} Z \circ g, \quad \operatorname{ker} J_{0}^{\prime}\left(\mathrm{U}_{q} \circ g\right)=\operatorname{ker} J_{0}^{\prime}(\mathrm{U}) \circ g, \quad$ for every $q \in \mathbb{H}^{3}, g \in P G L(2, \mathbb{C})$, it suffices to consider the case $U=\mathrm{U}$.

If $\varphi \in \mathcal{C}^{2}\left(\overline{\mathbb{R}}^{2}, \mathbb{R}^{3}\right)$ solves (1.4) then $J_{0}^{\prime}(\mathrm{U}) \varphi=0$, which means $\mathcal{P}\left(J_{0}^{\prime}(\mathrm{U}) \varphi\right)=0$ and $\left(J_{0}^{\prime}(\mathrm{U}) \varphi\right) \cdot \omega=0$. From Lemma 3.1 we infer that $\mathcal{P} \varphi$ solves $(3.14 \mathrm{a})$ and that $\varphi \cdot \omega$ solves (3.14b). Therefore, Lemmata 3.3,3.4 give the existence of $s, t, \alpha \in \mathbb{R}^{3}$ such that

$$
\mathcal{P} \varphi=s-(s \cdot \omega) \omega+t \wedge \omega, \quad \varphi \cdot \omega=\alpha \cdot\left(k \omega+e_{3}\right) .
$$

Thus $\varphi=\mathcal{P} \varphi+(\varphi \cdot \omega) \omega \in T_{\mathrm{U}} Z$ by (3.5), which concludes the proof.

\subsection{Further results on the operator $J_{0}^{\prime}(\mathrm{U})$}

To shorten notation we put

$$
H^{1}=H^{1}\left(\overline{\mathbb{R}}^{2}, \mathbb{R}^{3}\right) .
$$

Since integration by parts gives

$$
\int_{\mathbb{R}^{2}}-\operatorname{div}\left(\frac{\nabla \varphi}{\left(\omega_{3}+k\right)^{2}}\right) \cdot \psi d z=\int_{\mathbb{R}^{2}} \frac{\nabla \varphi \cdot \nabla \psi}{\left(\omega_{3}+k\right)^{2}} d z, \quad \varphi, \psi \in \mathcal{C}^{2}\left(\overline{\mathbb{R}}^{2}, \mathbb{R}^{3}\right),
$$

the quadratic form

$$
(\varphi, \psi) \mapsto \int_{\mathbb{R}^{2}} J_{0}^{\prime}(\mathrm{U}) \varphi \cdot \psi d z
$$

can be extended to a continuous bilinear form $H^{1} \times H^{1} \rightarrow \mathbb{R}$ via a density argument. It can be checked by direct computation (see also Remark 4.1) that the quadratic form in (3.19) is self-adjoint on $H^{1}$, that is,

$$
\int_{\mathbb{R}^{2}} J_{0}^{\prime}(\mathrm{U}) \varphi \cdot \psi d z=\int_{\mathbb{R}^{2}} J_{0}^{\prime}(\mathrm{U}) \psi \cdot \varphi d z \text { for any } \varphi, \psi \in H^{1} .
$$

Since $T_{\mathrm{U}} Z$ is a subspace of $L^{2}\left(\overline{\mathbb{R}}^{2}, \mathbb{R}^{3}\right) \equiv L^{2}\left(\mathbb{S}^{2}, \mathbb{R}^{3}\right)$, we are allowed to put

$$
T_{\mathrm{U}} Z^{\perp}:=\left\{f \in L^{2}\left(\overline{\mathbb{R}}^{2}, \mathbb{R}^{3}\right) \mid \int_{\mathbb{R}^{2}} f \cdot \tau \mu^{2} d z=0, \forall \tau \in T_{\mathrm{U}} Z\right\} .
$$

Moreover, we introduce on $L^{2}\left(\overline{\mathbb{R}}^{2}, \mathbb{R}^{3}\right)$ the equivalent scalar product

$$
(f, \psi)_{*}=\int_{\mathbb{R}^{2}} \frac{\mathcal{P} f \cdot \mathcal{P} \psi}{\left(\omega_{3}+k\right)^{2}} \mu^{2} d z+\int_{\mathbb{R}^{2}} \frac{(f \cdot \omega)(\psi \cdot \omega)}{\left(\omega_{3}+k\right)^{3}} \mu^{2} d z
$$


and the subspaces

$$
\begin{aligned}
T_{\mathrm{U}} Z_{*}^{\perp} & :=\left\{f \in L^{2}\left(\overline{\mathbb{R}}^{2}, \mathbb{R}^{3}\right) \mid(f, \tau)_{*}=0, \forall \tau \in T_{\mathrm{U}} Z\right\}, \\
N_{*} & :=\langle\omega\rangle_{*}^{\perp}=\left\{f \in L^{2}\left(\overline{\mathbb{R}}^{2}, \mathbb{R}^{3}\right) \mid(f, \omega)_{*}=0\right\} .
\end{aligned}
$$

We are in position to state the main result of this section.

Lemma 3.5 Let $q \in \mathbb{H}^{3}$. For any $v \in T_{\mathrm{U}} Z^{\perp}$, there exists $\varphi_{v} \in H^{1} \cap T_{\mathrm{U}} Z_{*}^{\perp} \cap N_{*}$ such that

$$
J_{0}^{\prime}\left(\mathrm{U}_{q}\right) \varphi_{v}=v \mu^{2} \quad \text { on } \mathbb{R}^{2} .
$$

If in addition $v \in \mathcal{C}^{m}\left(\overline{\mathbb{R}}^{2}, \mathbb{R}^{3}\right)$ for some $m \in(0,1)$, then $\varphi_{v} \in \mathcal{C}^{2+m}\left(\overline{\mathbb{R}}^{2}, \mathbb{R}^{3}\right)$.

In view of Lemma 3.1, we split the proof of Lemma 3.5 in few steps.

Lemma 3.6 Let $v \in T_{\mathrm{U}} Z^{\perp}$ be such that $v \cdot \omega \equiv 0$ on $\mathbb{R}^{2}$. There exists $\varphi \in H^{1} \cap T_{\mathrm{U}} Z_{*}^{\perp}$ such that $\varphi \cdot \omega \equiv 0$ on $\mathbb{R}^{2}$ and

$$
J_{0}^{\prime}(\mathrm{U}) \varphi=v \mu^{2} \quad \text { on } \mathbb{R}^{2} .
$$

Proof We introduce

$$
X:=\left\{\psi \in H^{1} \mid \psi \cdot \omega \equiv 0 \text { on } \mathbb{R}^{2}\right\} \cap T_{\mathrm{U}} Z_{*}^{\perp},
$$

which is a closed subspace of $H^{1}$. Notice that $\psi=\mathcal{P} \psi$ for any $\psi \in X$ and moreover

$$
\int_{\mathbb{R}^{2}} J_{0}^{\prime}(\mathrm{U}) \psi \cdot \psi d z=\int_{\mathbb{R}^{2}} \frac{|\nabla \psi|^{2}}{\left(\omega_{3}+k\right)^{2}} d z+2 \int_{\mathbb{R}^{2}}\left(\frac{(\psi \cdot i z \nabla \psi) \wedge \omega}{\left(\omega_{3}+k\right)^{3}}-\frac{|\psi|^{2}}{\left(\omega_{3}+k\right)^{2}}\right) \mu^{2} d z
$$

use (3.9) and a density argument. Next we put

$$
\lambda:=\inf _{\substack{\psi \in X \\ \psi \neq 0}} \frac{\int_{\mathbb{R}^{2}} J_{0}^{\prime}(\mathrm{U}) \psi \cdot \psi d z}{\int_{\mathbb{R}^{2}}\left(\omega_{3}+k\right)^{-2}|\psi|^{2} \mu^{2} d z},
$$

and notice that $\lambda \geq 0$ by Lemma 3.2. On the other hand, $\lambda$ is achieved by Rellich theorem. Thus $\lambda>0$, because of Lemma 3.3. It follows that the energy functional $I: X \rightarrow \mathbb{R}$,

$$
I(\psi)=\frac{1}{2} \int_{\mathbb{R}^{2}} J_{0}^{\prime}(\mathrm{U}) \psi \cdot \psi d z-\int_{\mathbb{R}^{2}} v \cdot \psi \mu^{2} d z
$$

is weakly lower semicontinuous and coercive. Thus its infimum is achieved by a function $\varphi \in X$ which satisfies

$$
\int_{\mathbb{R}^{2}} J_{0}^{\prime}(\mathrm{U}) \varphi \cdot \psi d z=\int_{\mathbb{R}^{2}} v \cdot \psi \mu^{2} d z, \quad \forall \psi \in X .
$$

If $\psi \in H^{1}$ we write

$$
\psi=\left(\mathcal{P} \psi^{\top}+\mathcal{P} \psi^{\perp}\right)+\eta \omega
$$


where $\eta=\psi \cdot \omega, \mathcal{P} \psi^{\top} \in T_{\mathrm{U}} Z=\operatorname{ker} J_{0}^{\prime}(U)$ is the orthogonal projection of $\mathcal{P} \psi=\psi-\eta \omega$ onto $T_{\mathrm{U}} Z$ in the scalar product $(\cdot, \cdot)_{*}$ and $\mathcal{P} \psi^{\perp}:=\psi-\mathcal{P} \psi^{\top}-\eta \omega \in X$. We use (3.20) and (3.10) to compute

$$
\begin{aligned}
& \int_{\mathbb{R}^{2}} J_{0}^{\prime}(\mathrm{U}) \varphi \cdot \mathcal{P} \psi^{\top} d z=\int_{\mathbb{R}^{2}} J_{0}^{\prime}(\mathrm{U}) \mathcal{P} \psi^{\top} \cdot \psi d z=0, \\
& \int_{\mathbb{R}^{2}} J_{0}^{\prime}(\mathrm{U}) \varphi \cdot(\eta \omega) d z=\int_{\mathbb{R}^{2}} \frac{\nabla(\varphi \cdot \omega) \cdot \nabla \eta}{\left(\omega_{3}+k\right)^{2}} d z-2 k \int_{\mathbb{R}^{2}} \frac{(\varphi \cdot \omega) \eta}{\left(\omega_{3}+k\right)^{3}} \mu^{2} d z=0,
\end{aligned}
$$

because $\varphi \cdot \omega \equiv 0$. Therefore, (3.23) gives

$$
\int_{\mathbb{R}^{2}} J_{0}^{\prime}(\mathrm{U}) \varphi \cdot \psi d z=\int_{\mathbb{R}^{2}} J_{0}^{\prime}(\mathrm{U}) \varphi \cdot \mathcal{P} \psi^{\perp} d z=\int_{\mathbb{R}^{2}} v \cdot \mathcal{P} \psi^{\perp} \mu^{2} d z=\int_{\mathbb{R}^{2}} v \cdot \psi \mu^{2} d z,
$$

as $v$ is orthogonal to $T_{\mathrm{U}} Z \ni \mathcal{P} \psi^{\top}$ and to $\eta \omega$ in $L^{2}\left(\overline{\mathbb{R}}^{2}, \mathbb{R}^{3}\right)$. We showed that $\varphi$ solves (3.22), and thus the proof is complete.

Lemma 3.7 Let $f \in H^{1}\left(\overline{\mathbb{R}}^{2}\right)$ be such that $f \omega \in T_{\mathrm{U}} Z^{\perp}$. There exists $\eta \in H^{1}\left(\overline{\mathbb{R}}^{2}\right)$ such that $\eta \omega \in H^{1} \cap T_{\mathrm{U}} Z_{*}^{\perp} \cap N_{*}$ and

$$
J_{0}^{\prime}(\mathrm{U})(\eta \omega)=f \omega \mu^{2} \text { on } \mathbb{R}^{2} .
$$

Proof We introduce the space

$$
Y:=\left\{\eta \in H^{1}\left(\overline{\mathbb{R}}^{2}\right) \mid \int_{\mathbb{R}^{2}} \frac{\eta}{\left(\omega_{3}+k\right)^{3}} \mu^{2} d z=\int_{\mathbb{R}^{2}} \frac{\eta(\tau \cdot \omega)}{\left(\omega_{3}+k\right)^{3}} \mu^{2} d z=0, \forall \tau \in T_{\mathrm{U}} Z\right\},
$$

so that $\eta \omega \in H^{1} \cap T_{\mathrm{U}} Z_{*}^{\perp} \cap N_{*}$ for any $\eta \in Y$, and the energy functional $I: Y \rightarrow \mathbb{R}$,

$$
\begin{aligned}
I(\varphi) & =\frac{1}{2} \int_{\mathbb{R}^{2}} J_{0}^{\prime}(\mathrm{U})(\eta \omega) \cdot(\eta \omega) d z-\int_{\mathbb{R}^{2}} f \eta \mu^{2} d z \\
& =\frac{1}{2} \int_{\mathbb{R}^{2}} \frac{|\nabla \eta|^{2}}{\left(\omega_{3}+k\right)^{2}} d z-k \int_{\mathbb{R}^{2}} \frac{|\eta|^{2}}{\left(\omega_{3}+k\right)^{3}} \mu^{2} d z-\int_{\mathbb{R}^{2}} \eta f \mu^{2} d z,
\end{aligned}
$$

compare with (3.10). The functional $I$ is weakly lower semicontinuous with respect to the $H^{1}\left(\overline{\mathbb{R}}^{2}\right)$ topology and coercive by Remark 3.2. Thus its infimum is achieved by a function $\eta \in Y$. To conclude, argue as in the proof of Lemma 3.6 to show that $\eta$ solves (3.24).

Proof of Lemma 3.5 Since $J_{0}^{\prime}\left(\mathrm{U}_{q}\right)=q_{3}^{-2} J_{0}^{\prime}(\mathrm{U})$, we can assume that $q=e_{3}$, that is, $\mathrm{U}_{q}=\mathrm{U}$. We take any $v \in T_{\mathrm{U}} Z^{\perp}$, and write

$$
v=\mathcal{P} v+(v \cdot \omega) \omega,
$$

where $\mathcal{P} v=v-(v \cdot \omega) \omega$, as before. Since $\mathcal{P} v \in T_{\mathrm{U}} Z^{\perp}$, by Lemma 3.6 there exists a unique $\hat{\varphi} \in H^{1} \cap T_{\mathrm{U}} Z_{*}^{\perp}$ such that $\hat{\varphi} \cdot \omega \equiv 0$ on $\mathbb{R}^{2}$ and

$$
\int_{\mathbb{R}^{2}} J_{0}^{\prime}(\mathrm{U}) \hat{\varphi} \cdot \psi d z=\int_{\mathbb{R}^{2}} \mathcal{P} v \cdot \psi \mu^{2} d z, \quad \text { for any } \psi \in H^{1} .
$$


Next, notice that $(v \cdot \omega) \omega \in T_{\mathrm{U}} Z^{\perp}$, so we can use Lemma 3.7 to find $\eta \in H^{1}\left(\overline{\mathbb{R}}^{2}\right)$ such that $\eta \omega \in H^{1} \cap T_{\mathrm{U}} Z_{*}^{\perp} \cap N_{*}$ solves

$$
\int_{\mathbb{R}^{2}} J_{0}^{\prime}(\mathrm{U})(\eta \omega) \cdot \psi d z=\int_{\mathbb{R}^{2}}(v \cdot \omega)(\psi \cdot \omega) \mu^{2} d z, \quad \text { for any } \psi \in H^{1} .
$$

The function $\varphi_{v}=\hat{\varphi}+\eta \omega$ solves (3.21).

To conclude the proof we have to show that if $v \in \mathcal{C}^{m}\left(\overline{\mathbb{R}}^{2}, \mathbb{R}^{3}\right)$ then $\varphi_{v} \in \mathcal{C}^{2+m}\left(\overline{\mathbb{R}}^{2}, \mathbb{R}^{3}\right)$. Since $\omega \in \mathcal{C}^{\infty}\left(\overline{\mathbb{R}}^{2}, \mathbb{R}^{3}\right)$ and $\omega_{3}+k$ is bounded and bounded away from zero, $\varphi_{v}$ solves a linear system of the form

$$
-\Delta \varphi_{v}=A(z) \varphi_{v}+B(z) \nabla \varphi_{v}+\mu^{2}\left(\omega_{3}+k\right)^{2} v,
$$

for certain smooth matrices on $\overline{\mathbb{R}}^{2}$. A standard bootstrap argument and Schauder regularity theory plainly imply that $\varphi_{v} \in \mathcal{C}_{l o c}^{2+m}\left(\mathbb{R}^{2}, \mathbb{R}^{3}\right)$. The function $z \mapsto \varphi_{v}\left(z^{-1}\right)$ satisfies a linear system of the same kind, hence $\varphi_{v} \in \mathcal{C}^{2+m}\left(\overline{\mathbb{R}}^{2}, \mathbb{R}^{3}\right)$, as desired.

\section{The perturbed problem}

In this section we perform the finite dimensional reduction and prove Theorems 1.2, 1.3. By the results in the Appendix, any critical point of the $\mathcal{C}^{2}$-functional $E_{\varepsilon}: \mathcal{C}^{2}\left(\overline{\mathbb{R}}^{2}, \mathbb{H}^{3}\right) \rightarrow \mathbb{R}$,

$$
E_{\varepsilon}(u):=\frac{1}{2} \int_{\mathbb{R}^{2}} u_{3}^{-2}|\nabla u|^{2} d z-k \int_{\mathbb{R}^{2}} u_{3}^{-2} e_{3} \cdot \partial_{x} u \wedge \partial_{y} u d z+2 \varepsilon V_{\phi}(u)=E_{0}(u)+2 \varepsilon V_{\phi}(u)
$$

(notice that $E_{0}=E_{\mathrm{hyp}}$, compare with (1.3)), solves

$$
\Delta u-2 u_{3}^{-1} G(\nabla u)=2 u_{3}^{-1}(k+\varepsilon \phi(u)) \partial_{x} u \wedge \partial_{y} u \text { on } \mathbb{R}^{2}
$$

and has mean curvature $(k+\varepsilon \phi)$, apart from a finite set of branch points.

Due to the action of the Möbius transformations and of the hyperbolic translations, for any $u \in \mathcal{C}^{2}\left(\overline{\mathbb{R}}^{2}, \mathbb{H}^{3}\right)$ we have the identities

$$
\begin{aligned}
& E_{\varepsilon}^{\prime}(u)\left(z^{h} \nabla u\right)=0, \quad E_{\varepsilon}^{\prime}(u)\left(i z^{h} \nabla u\right)=0, \quad \text { for } h=0,1,2, \quad \varepsilon \in \mathbb{R}, \\
& E_{0}^{\prime}(u) e_{1}=0, \quad E_{0}^{\prime}(u) e_{2}=0, \quad E_{0}^{\prime}(u) u=0 .
\end{aligned}
$$

Now we prove that

$$
E_{\varepsilon}\left(\mathrm{U}_{q}\right)=E_{0}(\mathrm{U})-2 \varepsilon F_{k}^{\phi}(q)
$$

where $F_{k}^{\phi}$ is the Melnikov-type function in (1.5). The above mentioned invariances give $E_{0}\left(\mathrm{U}_{q}\right)=E_{0}(\mathrm{U})$. Since the hyperbolic ball $B_{\rho_{k}}^{\mathbb{H}}(q)$ coincides with the Euclidean ball of radius $q_{3} r_{k}$ about the point $q^{k}:=\left(q_{1}, q_{2}, k r_{k} q_{3}\right)$, the divergence theorem gives

$$
F_{k}^{\phi}(q)=\int_{B_{\rho_{k}}^{\mathbb{H}}(q)} \phi(p) d \mathbb{H}_{p}^{3}=\int_{B_{q_{3} r_{k}}\left(q^{k}\right)} p_{3}^{-3} \phi(p) d p=\int_{\partial B_{q_{3} r_{k}}\left(q^{k}\right)} Q_{\phi}(p) \cdot v_{p} .
$$

Here $Q_{\phi} \in \mathcal{C}^{1}\left(\mathbb{R}_{+}^{3}, \mathbb{R}^{3}\right)$ is any vectorfield such that $\operatorname{div} Q_{\phi}(p)=p_{3}^{-3} \phi(p)$ and $v_{p}$ is the outer normal to $\partial B_{q_{3} r_{k}}\left(q^{k}\right)$ at $p$. The function $\mathrm{U}_{q}$ in (3.1) parameterizes the Euclidean sphere 
$\partial B q_{3} r_{k}\left(q^{k}\right)$. Since $\partial_{x} \mathrm{U}_{q} \wedge \partial_{y} \mathrm{U}_{q}$ is inward-pointing, we have

$$
F_{k}^{\phi}(q)=-\int_{\mathbb{R}^{2}} Q_{\phi}(p) \cdot \partial_{x} \mathrm{U}_{q} \wedge \partial_{y} \mathrm{U}_{q} d z=-V_{\phi}\left(\mathrm{U}_{q}\right),
$$

and (4.3) is proved. Before going further, let us show that the existence of critical points for $F_{k}^{\phi}$ is a necessary condition for the conclusion in Theorem 1.2.

Theorem 4.1 Let $k>1, \phi \in \mathcal{C}^{1}\left(\mathbb{H}^{3}\right)$. Assume that there exist sequences $\varepsilon_{h} \subset \mathbb{R} \backslash\{0\}$, $\varepsilon_{h} \rightarrow 0, u^{h} \in \mathcal{C}^{2}\left(\overline{\mathbb{R}}^{2}, \mathbb{H}^{3}\right)$ and a point $q \in \mathbb{H}^{3}$ such that $u_{h}$ solves $\left(\mathcal{P}_{\varepsilon_{h}}\right)$, and $u^{h} \rightarrow \mathrm{U}_{q}$ in $\mathcal{C}^{1}\left(\overline{\mathbb{R}}^{2}, \mathbb{H}^{3}\right)$. Then $q$ is a stationary point for $F_{k}^{\phi}$.

Proof The function $u^{h}$ is a stationary point for the energy functional $E_{\varepsilon_{h}}=E_{0}+2 \varepsilon_{h} V_{\phi}$. From (4.2) we have $V_{\phi}^{\prime}\left(u^{h}\right) e_{j}=0$ for $j=1,2$ and $V_{\phi}^{\prime}\left(u^{h}\right) u^{h}=0$. We can plainly pass to the limit to obtain $V_{\phi}^{\prime}\left(\mathrm{U}_{q}\right) e_{j}=0$ for $j=1,2$ and $V_{\phi}^{\prime}\left(\mathrm{U}_{q}\right) \mathrm{U}_{q}=0$. To conclude, use (4.4) and recall that $\partial_{q_{j}} \mathrm{U}_{q}=e_{j}$ for $j=1,2$, and $\partial_{q_{3}} \mathrm{U}_{q}=\mathrm{U}=q_{3}^{-1}\left(\mathrm{U}_{q}-q_{1} e_{1}-q_{2} e_{2}\right)$.

Now we fix $m \in(0,1)$. The operator $J_{\varepsilon}: \mathcal{C}^{2+m}\left(\overline{\mathbb{R}}^{2}, \mathbb{H}^{3}\right) \rightarrow \mathcal{C}^{m}\left(\overline{\mathbb{R}}^{2}, \mathbb{R}^{3}\right)$ defined by

$$
J_{\varepsilon}(u)=-\operatorname{div}\left(u_{3}^{-2} \nabla u\right)-u_{3}^{-3}|\nabla u|^{2} e_{3}+2(k+\varepsilon \phi) u_{3}^{-3} \partial_{x} u \wedge \partial_{y} u,
$$

is related to the differential of $E_{\varepsilon}$ via the identity

$$
E_{\varepsilon}^{\prime}(u) \varphi=\int_{\mathbb{R}^{2}} J_{\varepsilon}(u) \cdot \varphi d z, \quad u \in \mathcal{C}^{m}\left(\overline{\mathbb{R}}^{2}, \mathbb{H}^{3}\right), \varphi \in \mathcal{C}^{m}\left(\overline{\mathbb{R}}^{2}, \mathbb{R}^{3}\right)
$$

Remark 4.1 Since $E_{\varepsilon}$ is of class $\mathcal{C}^{2}$ and

$$
E_{\varepsilon}^{\prime \prime}(u)[\varphi, \psi]=\int_{\mathbb{R}^{2}} J_{\varepsilon}^{\prime}(u) \psi \cdot \varphi d z
$$

then the quadratic form in the right hand side is a self-adjoint form on $H^{1}$.

We are in position to state and prove the next Lemma, which is the main step towards the proofs of Theorems 1.2, 1.3.

Lemma 4.1 (Dimension reduction) Let $\Omega \Subset \mathbb{H}^{3}$ be an open set. There exist $\hat{\varepsilon}>0$ and $a$ unique $\mathcal{C}^{1}$-map

$$
[-\hat{\varepsilon}, \hat{\varepsilon}] \times \bar{\Omega} \rightarrow \mathcal{C}^{2+m}\left(\overline{\mathbb{R}}^{2}, \mathbb{H}^{3}\right), \quad(\varepsilon, q) \mapsto u_{q}^{\varepsilon},
$$

such that the following facts hold:

(i) $u_{q}^{\varepsilon}$ parameterizes an embedded $\mathbb{S}^{2}$-type surface, and $u_{q}^{0}=\mathrm{U}_{q}$;

(ii) $u_{q}^{\varepsilon}-\mathrm{U}_{q} \in T_{\mathrm{U}} Z^{\perp} \cap \mathcal{C}^{2+m}\left(\overline{\mathbb{R}}^{2}, \mathbb{R}^{3}\right)$ and $E_{\varepsilon}^{\prime}\left(u_{q}^{\varepsilon}\right) \varphi=0$ for any $\varphi \in T_{\mathrm{U}} Z^{\perp} \cap \mathcal{C}^{0}\left(\overline{\mathbb{R}}^{2}, \mathbb{R}^{3}\right)$;

(iii) for any $\varepsilon \in[-\hat{\varepsilon}, \hat{\varepsilon}]$, the manifold $\left\{u_{q}^{\varepsilon} \mid q \in \Omega\right\}$ is a natural constraint for $E_{\varepsilon}$, that is, if $\nabla_{q} E_{\varepsilon}\left(u_{q^{\varepsilon}}^{\varepsilon}\right)=0$ for some $q^{\varepsilon} \in \Omega$, then $u_{q^{\varepsilon}}^{\varepsilon}$ is a $(k+\varepsilon \phi)$-bubble;

(iv) $\left\|E_{\varepsilon}\left(u_{q}^{\varepsilon}\right)-E_{\varepsilon}\left(\mathrm{U}_{q}\right)\right\|_{\mathcal{C}^{1}(\bar{\Omega})}=o(\varepsilon)$ as $\varepsilon \rightarrow 0$, uniformly on $\bar{\Omega}$. 
Proof To shorten the notation, we put $\mathcal{C}^{m}:=\mathcal{C}^{m}\left(\overline{\mathbb{R}}^{2}, \mathbb{R}^{3}\right)$. For $s>0$ and $\delta>0$ we write $\Omega_{s}:=\left\{p \in \mathbb{H}^{3} \mid \operatorname{dist}(p, \Omega)<s\right\}$, and $\mathcal{U}_{\delta}:=\left\{v \in \mathcal{C}^{2+m}|| v(z) \mid<\delta\right.$ for every $\left.z \in \mathbb{R}^{2}\right\}$. We fix $s$ and $\delta=\delta(s)$ such that $\bar{\Omega}_{2 s} \subset \mathbb{H}^{3}$ and $\left(\mathrm{U}_{q}+v\right) \cdot e_{3}>0$ for $q \in \Omega_{2 s}, v \in \mathcal{U}_{\delta}$.

We define

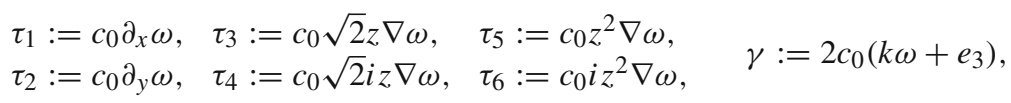

where $c_{0}:=\sqrt{\frac{3}{2^{4} \pi}}$ is a normalization constant. Thanks to (3.3), (3.5), we have

$$
T_{\mathrm{U}} Z=\left\langle\tau_{1}, \ldots \tau_{6}\right\rangle \oplus\left\{(\alpha \cdot \gamma) \omega \mid \alpha \in \mathbb{R}^{3}\right\} .
$$

Trivially, $\tau_{j} \cdot \omega \equiv 0$ on $\mathbb{R}^{2}$. Elementary computations give

$$
\int_{\mathbb{R}^{2}} \tau_{i} \cdot \tau_{j} \mu^{2} d z=\delta_{i j}, \quad \int_{\mathbb{R}^{2}} \gamma_{h} \gamma_{\ell} \mu^{2} d z=0 \text { if } h \neq \ell,
$$

for $i, j \in\{1, \ldots, 6\}, h, \ell \in\{1,2,3\}$, and moreover

$$
\int_{\mathbb{R}^{2}} \gamma_{1}^{2} \mu^{2} d z=\int_{\mathbb{R}^{2}} \gamma_{2}^{2} \mu^{2} d z=k^{2}, \quad \int_{\mathbb{R}^{2}} \gamma_{3}^{2} \mu^{2} d z=k^{2}+3 .
$$

Construction of $\mathbf{u}_{\mathbf{q}}^{\varepsilon}$ satisfying $\left.\left.\boldsymbol{i}\right), \mathbf{i i}\right)$. By our choices of $s$ and $\delta$, the functions

$$
\begin{aligned}
& \mathcal{F}_{1}(\varepsilon, q ; v, \xi, \alpha):=\mu^{-2} J_{\varepsilon}\left(\mathrm{U}_{q}+v\right)-\sum_{j=1}^{6} \xi_{j} \tau_{j}-(\alpha \cdot \gamma) \omega \in \mathcal{C}^{m}, \\
& \mathcal{F}_{2}(\varepsilon, q ; v, \xi, \alpha):=\left(\int_{\mathbb{R}^{2}} v \cdot \tau_{1} \mu^{2} d z, \ldots, \int_{\mathbb{R}^{2}} v \cdot \tau_{6} \mu^{2} d z ; \int_{\mathbb{R}^{2}} \gamma(v \cdot \omega) \mu^{2} d z\right) \in \mathbb{R}^{6} \times \mathbb{R}^{3},
\end{aligned}
$$

are well defined and continuously differentiable on $\mathbb{R} \times \Omega_{2 s} \times \mathcal{U}_{\delta} \times\left(\mathbb{R}^{6} \times \mathbb{R}^{3}\right)$. Thus

$$
\mathcal{F}:=\left(\mathcal{F}_{1}, \mathcal{F}_{2}\right): \mathbb{R} \times \Omega_{2 s} \times \mathcal{U}_{\delta} \times\left(\mathbb{R}^{6} \times \mathbb{R}^{3}\right) \rightarrow \mathcal{C}^{m} \times\left(\mathbb{R}^{6} \times \mathbb{R}^{3}\right)
$$

is of class $\mathcal{C}^{1}$ on its domain. Notice that $\mathcal{F}(0, q ; 0,0,0)=0$ for every $q \in \Omega_{2 s}$ because $J_{0}\left(\mathrm{U}_{q}\right)=0$. Now we solve the equation $\mathcal{F}(\varepsilon, q ; v, \xi, \alpha)=0$ in a neighborhood of $(0, q ; 0,0,0)$ via the implicit function theorem. Let

$$
\mathcal{L}:=\left(\mathcal{L}_{1}, \mathcal{L}_{2}\right): \mathcal{C}^{2+m} \times\left(\mathbb{R}^{6} \times \mathbb{R}^{3}\right) \rightarrow \mathcal{C}^{m} \times\left(\mathbb{R}^{6} \times \mathbb{R}^{3}\right)
$$

given by

$$
\begin{aligned}
& \mathcal{L}_{1}(\varphi ; \zeta, \beta):=\mu^{-2} J_{0}^{\prime}\left(\mathrm{U}_{q}\right) \varphi-\sum_{j=1}^{6} \zeta_{j} \tau_{j}-(\beta \cdot \gamma) \omega \\
& \mathcal{L}_{2}(\varphi ; \zeta, \beta):=\mathcal{L}_{2}(\varphi)=\left(\int_{\mathbb{R}^{2}} \varphi \cdot \tau_{1} \mu^{2} d z, \ldots, \int_{\mathbb{R}^{2}} \varphi \cdot \tau_{6} \mu^{2} d z ; \int_{\mathbb{R}^{2}} \gamma(\varphi \cdot \omega) \mu^{2} d z\right),
\end{aligned}
$$

so that $\mathcal{L}=\left(\mathcal{L}_{1}, \mathcal{L}_{2}\right)$ is the differential of $\mathcal{F}(0, q ; \cdot, \cdot, \cdot)$ evaluated in $(\nu, \xi, \alpha)=(0,0,0)$.

To prove that $\mathcal{L}$ is injective we assume that $\mathcal{L}(\varphi, \zeta, \beta)=0$ and put

$$
v=\mu^{-2} J_{0}^{\prime}\left(\mathrm{U}_{q}\right) \varphi \in T_{\mathrm{U}} Z \text {. }
$$


From (3.20) we find

$$
\int_{\mathbb{R}^{2}}|v|^{2} \mu^{2} d z=\int_{\mathbb{R}^{2}}\left(\mu^{-2} J_{0}^{\prime}\left(\mathrm{U}_{q}\right) \varphi\right) \cdot v \mu^{2} d z=\int_{\mathbb{R}^{2}} J_{0}^{\prime}\left(\mathrm{U}_{q}\right) \varphi \cdot v d z=\int_{\mathbb{R}^{2}} J_{0}^{\prime}\left(\mathrm{U}_{q}\right) v \cdot \varphi d z=0,
$$

which implies $J_{0}^{\prime}\left(\mathrm{U}_{q}\right) \varphi=0$, that is, $\varphi \in T_{\mathrm{U}} Z$. On the other hand, $\varphi \in T_{\mathrm{U}} Z^{\perp}$ because $\mathcal{L}_{2}(\varphi)=0$. Thus $\varphi=0$ and therefore also $\beta=\zeta=0$.

To prove that $\mathcal{L}$ is surjective fix $v \in \mathcal{C}^{m}$ and $(\theta, b) \in \mathbb{R}^{6} \times \mathbb{R}^{3}$. We have to find $\varphi \in \mathcal{C}^{2+m}$ and $(\zeta, \beta) \in \mathbb{R}^{6} \times \mathbb{R}^{3}$ such that $\mathcal{L}_{1}(\varphi ; \zeta, \beta)=v$ and $\mathcal{L}_{2}(\varphi)=(\theta, b)$. To this goal we introduce the minimal distance projection

$$
P^{\top}: L^{2}\left(\overline{\mathbb{R}}^{2}, \mathbb{R}^{3}\right) \rightarrow T_{\mathrm{U}} Z, \quad w \mapsto P^{\top} w,
$$

so that $\mathcal{L}_{2}(w)$ is uniquely determined by $P^{\top} w$, and vice-versa. We find $\zeta_{j}$ and $\beta$ so that

$$
\sum_{j=1}^{6} \zeta_{j} \tau_{j}+(\beta \cdot \gamma) \omega=-P^{\top} v
$$

Then, we use Lemma 3.5 to find $\widehat{\varphi} \in \mathcal{C}^{2+m} \cap T_{\mathrm{U}} Z_{*}^{\perp} \cap N_{*}$ such that

$$
J_{0}^{\prime}\left(\mathrm{U}_{q}\right) \widehat{\varphi}=\left(v-P^{\top} v\right) \mu^{2} .
$$

Finally, we take the unique tangent direction $\varphi^{\top} \in T_{\mathrm{U}} Z$ such that $\mathcal{L}_{2}\left(\varphi^{\top}\right)=(\theta, b)-\mathcal{L}_{2}(\widehat{\varphi})$. The triple $\left(\varphi^{\top}+\widehat{\varphi} ; \zeta, \beta\right)$ satisfies $\mathcal{L}\left(\varphi^{\top}+\widehat{\varphi} ; \zeta, \beta\right)=(v ; \theta, b)$ and surjectivity is proved. We are in the position to apply the implicit function theorem to $\mathcal{F}$, for any fixed $q \in \Omega_{2 s}$. In fact, thanks to a standard compactness argument, we get that there exist $\varepsilon^{\prime}>0$ and uniquely determined $\mathcal{C}^{1}$ functions

$$
\begin{array}{lll}
v:\left(-\varepsilon^{\prime}, \varepsilon^{\prime}\right) \times \Omega_{s} \rightarrow \mathcal{U}_{\delta} & \alpha:\left(-\varepsilon^{\prime}, \varepsilon^{\prime}\right) \times \Omega_{s} \rightarrow \mathbb{R}^{3} & \xi:\left(-\varepsilon^{\prime}, \varepsilon^{\prime}\right) \times \Omega_{s} \rightarrow \mathbb{R}^{6} \\
v:(\varepsilon, q) \mapsto v_{q}^{\varepsilon} & \alpha:(\varepsilon, q) \mapsto \alpha^{\varepsilon}(q) & \xi:(\varepsilon, q) \mapsto \xi^{\varepsilon}(q)
\end{array}
$$

such that

$$
v_{q}^{0} \equiv 0, \quad \alpha^{0}(q)=0, \quad \xi^{0}(q)=0, \quad \mathcal{F}\left(\varepsilon, q ; v_{q}^{\varepsilon}, \xi^{\varepsilon}(q), \alpha^{\varepsilon}(q)\right)=0 .
$$

By (4.7), the $\mathcal{C}^{1}$ function $\left(-\varepsilon^{\prime}, \varepsilon^{\prime}\right) \times \Omega_{s} \rightarrow \mathcal{C}^{2+m}\left(\overline{\mathbb{R}}^{2}, \mathbb{H}^{3}\right)$,

$$
(\varepsilon, q) \mapsto u_{q}^{\varepsilon}:=\mathrm{U}_{q}+v_{q}^{\varepsilon}=\left(q_{3} \mathrm{U}+q_{1} e_{1}+q_{2} e_{2}\right)+v_{q}^{\varepsilon},
$$

satisfies $i$ ), if $\varepsilon^{\prime}$ is small enough. Further, using (4.5) (see also Lemma A.1) we rewrite the last identity in (4.7) as

$$
\begin{aligned}
E_{\varepsilon}^{\prime}\left(u_{q}^{\varepsilon}\right) \varphi & =\int_{\mathbb{R}^{2}} J_{\varepsilon}^{\prime}\left(\mathrm{U}_{q}+v_{q}^{\varepsilon}\right) \cdot \varphi d z \\
& =\sum_{j=1}^{6} \xi_{j}^{\varepsilon}(q) \int_{\mathbb{R}^{2}} \tau_{j} \cdot \varphi \mu^{2} d z+\int_{\mathbb{R}^{2}}\left(\alpha^{\varepsilon}(q) \cdot \gamma\right)(\omega \cdot \varphi) \mu^{2} d z \quad \forall \varphi \in \mathcal{C}^{0}, \\
\int_{\mathbb{R}^{2}} v_{q}^{\varepsilon} \cdot \tau_{j} \mu^{2} d z & =0, \quad \forall j \in\{1, \ldots, 6\}, \quad \int_{\mathbb{R}^{2}} \gamma_{\ell}\left(v_{q}^{\varepsilon} \cdot \omega\right) \mu^{2} d z=0, \quad \forall \ell \in\{1,2,3\} .
\end{aligned}
$$

In particular, claim $i i$ ) holds true. 
Proof of iii). As a straightforward consequence of (4.8) we have that

$$
\int_{\mathbb{R}^{2}} \partial_{q_{i}} v_{q}^{\varepsilon} \cdot \tau_{j} \mu^{2} d z=0, \quad \int_{\mathbb{R}^{2}} \gamma_{\ell}\left(\partial_{q_{i}} v_{q}^{\varepsilon} \cdot \omega\right) \mu^{2} d z=0,
$$

hence $E_{\varepsilon}^{\prime}\left(u_{q}^{\varepsilon}\right) \partial_{q_{i}} v_{q}^{\varepsilon}=0$ for any $i=1,2,3$. We infer the identities

$$
\begin{aligned}
& \partial_{q_{i}} E_{\varepsilon}\left(u_{q}^{\varepsilon}\right)=E_{\varepsilon}^{\prime}\left(u_{q}^{\varepsilon}\right)\left(e_{i}+\partial_{q_{i}} v_{q}^{\varepsilon}\right)=E_{\varepsilon}^{\prime}\left(u_{q}^{\varepsilon}\right) e_{i}, \quad i=1,2, \\
& \partial_{q_{3}} E_{\varepsilon}\left(u_{q}^{\varepsilon}\right)=E_{\varepsilon}^{\prime}\left(u_{q}^{\varepsilon}\right)\left(\mathrm{U}+\partial_{q_{3}} v_{q}^{\varepsilon}\right)=E_{\varepsilon}^{\prime}\left(u_{q}^{\varepsilon}\right) \mathrm{U} .
\end{aligned}
$$

Now, from (2.4), (4.6) and (4.8) we find

$$
\begin{aligned}
2 c_{0} e_{1} & =\tau_{1}-\tau_{5}+k^{-1} \gamma_{1} \omega, \quad 2 c_{0} e_{2}=\tau_{2}+\tau_{6}+k^{-1} \gamma_{2} \omega, \quad 2 c_{0} \mathrm{U}=k r_{k}\left(\sqrt{2} \tau_{3}+k^{-1} \gamma_{3} \omega\right), \\
E_{\varepsilon}^{\prime}\left(u_{q}^{\varepsilon}\right) \tau_{j} & =\xi_{j}^{\varepsilon}(q), \quad E_{\varepsilon}^{\prime}\left(u_{q}^{\varepsilon}\right)\left(\gamma_{\ell} \omega\right)=\left(k^{2}+3 \delta_{\ell 3}\right) \alpha_{\ell}^{\varepsilon}(q),
\end{aligned}
$$

for any $j=1, \ldots, 6, \ell=1,2,3$. Thus by (4.9) we get

$$
2 c_{0} \nabla_{q} E_{\varepsilon}\left(u_{q}^{\varepsilon}\right)=M_{k} \xi^{\varepsilon}(q)+\Theta_{k} \alpha^{\varepsilon}(q),
$$

where $M_{k}$ and $\Theta_{k}$ are constant matrices, namely

$$
M_{k}=\left(\begin{array}{cccccc}
1 & 0 & 0 & 0 & -1 & 0 \\
0 & 1 & 0 & 0 & 0 & -1 \\
0 & 0 & \sqrt{2} k r_{k} & 0 & 0 & 0
\end{array}\right), \quad \Theta_{k}=\left(\begin{array}{ccc}
k & 0 & 0 \\
0 & k & 0 \\
0 & 0 & \left(k^{2}+3\right) r_{k}
\end{array}\right) .
$$

On the other hand, from (4.1) and using $\nabla \mathrm{U}_{q}=r_{k} q_{3} \nabla \omega$ we obtain

$$
-q_{3} r_{k} \xi_{j}^{\varepsilon}(q)=E_{\varepsilon}^{\prime}\left(u_{q}^{\varepsilon}\right)\left(\tau_{j}^{\varepsilon}(q)\right)
$$

where, in the spirit of (4.6), we have put

$$
\begin{array}{lll}
\tau_{1}^{\varepsilon}(q):=c_{0} \partial_{x} v_{q}^{\varepsilon}, & \tau_{3}^{\varepsilon}(q):=c_{0} \sqrt{2} z \nabla v_{q}^{\varepsilon}, & \tau_{5}^{\varepsilon}(q):=c_{0} z^{2} \nabla v_{q}^{\varepsilon}, \\
\tau_{2}^{\varepsilon}(q):=c_{0} \partial_{y} v_{q}^{\varepsilon}, & \tau_{4}^{\varepsilon}(q):=c_{0} \sqrt{2} i z \nabla v_{q}^{\varepsilon}, & \tau_{6}^{\varepsilon}(q):=c_{0} i z^{2} \nabla v_{q}^{\varepsilon}
\end{array}
$$

Notice that

$$
\int_{\mathbb{R}^{2}}\left|\tau_{j}^{\varepsilon}(q)\right|^{2} \mu^{2} d z \leq 2 \int_{\mathbb{R}^{2}}\left|\nabla_{z} v_{q}^{\varepsilon}\right|^{2} \mu d z \leq 2\left\|v_{q}^{\varepsilon}\right\|_{\mathcal{C}^{1}}^{2} \int_{\mathbb{R}^{2}} \mu^{3} d z=o(1),
$$

as $\varepsilon \rightarrow 0$, uniformly on $\bar{\Omega}$, see (2.3).

For the sake of clarity, we make now some explicit computations. We denote by $\sigma_{\ell h}$ the entries of the $3 \times 6$ constant matrix $\Theta_{k}^{-1} M_{k}$, and introduce the $6 \times 6$ matrix $A^{\varepsilon}(q)=$ $\left(a_{j h}^{\varepsilon}(q)\right)_{j, h=1, \ldots, 6}$, whose entries are given by

$$
a_{j h}^{\varepsilon}(q)=\int_{\mathbb{R}^{2}} \tau_{h} \cdot \tau_{j}^{\varepsilon}(q) \mu^{2} d z-\sum_{\ell=1}^{3} \sigma_{\ell h} \int_{\mathbb{R}^{2}} \gamma_{\ell}\left(\omega \cdot \tau_{j}^{\varepsilon}(q)\right) \mu^{2} d z .
$$

Since $\tau_{j}^{\varepsilon} \mu \rightarrow 0$ in $L^{2}\left(\mathbb{R}^{2}, \mathbb{R}^{3}\right)$ by (4.12), then $A^{\varepsilon} \rightarrow 0$ uniformly on compact subsets of $\left(-\varepsilon^{\prime}, \varepsilon^{\prime}\right) \times \Omega_{s}$. In particular, if $\hat{\varepsilon} \in\left(0, \varepsilon^{\prime}\right)$ is small enough, then the determinant of the $6 \times 6$ matrix $\left(A^{\varepsilon}(q)+q_{3} r_{k}\right.$ Id) is uniformly bounded away from 0 on $[-\hat{\varepsilon}, \hat{\varepsilon}] \times \bar{\Omega}$.

Assume that $\nabla_{q} E_{\varepsilon}\left(u_{q^{\varepsilon}}^{\varepsilon}\right)=0$ for some $\varepsilon \in[-\hat{\varepsilon}, \hat{\varepsilon}], q^{\varepsilon} \in \Omega$. From (4.10) we obtain $\alpha^{\varepsilon}\left(q^{\varepsilon}\right)=-\Theta_{k}^{-1} M_{k} \xi^{\varepsilon}\left(q^{\varepsilon}\right)$. Thus (4.8) and (4.11) give

$$
-q_{3}^{\varepsilon} r_{k} \xi^{\varepsilon}\left(q^{\varepsilon}\right)=A^{\varepsilon}\left(q^{\varepsilon}\right) \xi^{\varepsilon}\left(q^{\varepsilon}\right),
$$


and hence $\xi^{\varepsilon}\left(q^{\varepsilon}\right)=0$, because the matrix $\left(A^{\varepsilon}\left(q^{\varepsilon}\right)+q_{3}^{\varepsilon} r_{k} \mathrm{Id}\right)$ is invertible. But then (4.10) and $\nabla_{q} E_{\varepsilon}\left(u_{q^{\varepsilon}}^{\varepsilon}\right)=0$ imply that $\alpha^{\varepsilon}\left(q^{\varepsilon}\right)=0$ as well, hence $E^{\prime}\left(u_{q^{\varepsilon}}^{\varepsilon}\right)=0$ by (4.8).

Proof of iv). The function $(\varepsilon, q) \mapsto \nu_{q}^{\varepsilon}$ is of class $\mathcal{C}^{1}$, and in particular $\partial_{\varepsilon} v_{q}^{\varepsilon}$ is uniformly bounded in $\mathcal{C}^{2}$ for $(\varepsilon, q) \in[-\hat{\varepsilon}, \hat{\varepsilon}] \times \bar{\Omega}$. Thus Taylor expansion formula for

$$
\varepsilon \mapsto E_{\varepsilon}\left(u_{q}^{\varepsilon}\right)-E_{\varepsilon}\left(\mathrm{U}_{q}\right)=E_{0}\left(u_{q}^{\varepsilon}\right)-E_{0}\left(\mathrm{U}_{q}\right)+2 \varepsilon\left(V_{\phi}\left(u_{q}^{\varepsilon}\right)-V_{\phi}\left(\mathrm{U}_{q}\right)\right)
$$

gives $E_{\varepsilon}\left(u_{q}^{\varepsilon}\right)-E_{\varepsilon}\left(\mathrm{U}_{q}\right)=o(\varepsilon)$ as $\varepsilon \rightarrow 0$, uniformly on $\bar{\Omega}$.

Now we estimate $\nabla_{q}\left(E_{\varepsilon}\left(u_{q}^{\varepsilon}\right)-E_{\varepsilon}\left(\mathrm{U}_{q}\right)\right)$. We use (4.2), (4.9) to obtain, for $j=1,2$,

$$
\begin{aligned}
\partial_{q_{j}}\left(E_{\varepsilon}\left(u_{q}^{\varepsilon}\right)-E_{\varepsilon}\left(\mathrm{U}_{q}\right)\right) & =\left(E_{0}^{\prime}\left(u_{q}^{\varepsilon}\right) e_{j}-E_{0}^{\prime}\left(\mathrm{U}_{q}\right) e_{j}\right)+2 \varepsilon\left(V_{\phi}^{\prime}\left(u_{q}^{\varepsilon}\right) e_{j}-V_{\phi}^{\prime}\left(\mathrm{U}_{q}\right) e_{j}\right) \\
& =2 \varepsilon\left(V_{\phi}^{\prime}\left(u_{q}^{\varepsilon}\right) e_{j}-V_{\phi}^{\prime}\left(\mathrm{U}_{q}\right) e_{j}\right)=o(\varepsilon),
\end{aligned}
$$

because $\left\|u_{q}^{\varepsilon}-\mathrm{U}_{q}\right\|_{\mathcal{C}^{2+m}}=o(1)$ and $V_{\phi}$ is a $\mathcal{C}^{1}$-functional.

To handle the derivative with respect to $q_{3}$ we first argue as before to get

$$
\begin{aligned}
\partial_{q_{3}}\left(E_{\varepsilon}\left(u_{q}^{\varepsilon}\right)-E_{\varepsilon}\left(\mathrm{U}_{q}\right)\right) & =\left(E_{0}^{\prime}\left(u_{q}^{\varepsilon}\right) \mathrm{U}-E_{0}^{\prime}\left(\mathrm{U}_{q}\right) \mathrm{U}\right)+2 \varepsilon\left(V_{\phi}^{\prime}\left(u_{q}^{\varepsilon}\right) \mathrm{U}-V_{\phi}^{\prime}\left(\mathrm{U}_{q}\right) \mathrm{U}\right) \\
& =E_{0}^{\prime}\left(u_{q}^{\varepsilon}\right) \mathrm{U}+o(\varepsilon),
\end{aligned}
$$

uniformly on $\bar{\Omega}$. Next, from $q_{3} \mathrm{U}=u_{q}^{\varepsilon}-\left(q_{1} e_{1}+q_{2} e_{2}\right)-v_{q}^{\varepsilon}$ and (4.2) we obtain

$$
\begin{aligned}
q_{3} E_{0}^{\prime}\left(u_{q}^{\varepsilon}\right) \mathrm{U} & =E_{0}^{\prime}\left(u_{q}^{\varepsilon}\right)\left(u_{q}^{\varepsilon}-\left(q_{1} e_{1}+q_{2} e_{2}\right)-v_{q}^{\varepsilon}\right) \\
& =-E_{0}^{\prime}\left(u_{q}^{\varepsilon}\right) v_{q}^{\varepsilon}=-E_{\varepsilon}^{\prime}\left(u_{q}^{\varepsilon}\right) v_{q}^{\varepsilon}+2 \varepsilon V_{\phi}^{\prime}\left(u_{q}^{\varepsilon}\right) v_{q}^{\varepsilon}=2 \varepsilon V_{\phi}^{\prime}\left(u_{q}^{\varepsilon}\right) v_{q}^{\varepsilon}
\end{aligned}
$$

because of (4.8). Since $v_{q}^{\varepsilon} \rightarrow 0$ in $\mathcal{C}^{2+m}$ we infer that $E_{0}^{\prime}\left(u_{q}^{\varepsilon}\right) u_{q}^{\varepsilon}=o(\varepsilon)$ uniformly on $\bar{\Omega}$ as $\varepsilon \rightarrow 0$, which concludes the proof.

Proof of Theorem 1.2 Take an open set $\Omega \Subset \mathbb{R}_{+}^{3}$ containing the closure of $A$, let $u_{q}^{\varepsilon}$ be the function given by Lemma 4.1 and notice that, by (4.4), $E_{\varepsilon}\left(\mathrm{U}_{q}\right)=E_{0}\left(\mathrm{U}_{q}\right)-2 \varepsilon F_{k}^{\phi}(q)$. Thus for $\varepsilon \in[-\hat{\varepsilon}, \hat{\varepsilon}], \varepsilon \neq 0$ we can estimate

$$
\left\|\frac{1}{2 \varepsilon}\left(E_{\varepsilon}\left(u_{q}^{\varepsilon}\right)-E_{0}\left(\mathrm{U}_{q}\right)\right)+F_{k}^{\phi}(q)\right\|_{\mathcal{C}^{1}(\bar{A})}=\frac{1}{2|\varepsilon|}\left\|E_{\varepsilon}\left(u_{q}^{\varepsilon}\right)-E_{\varepsilon}\left(\mathrm{U}_{q}\right)\right\|_{\mathcal{C}^{1}(\bar{A})}=o(1),
$$

uniformly on $\bar{\Omega}$ by $i v$ ) in Lemma 4.1. Recalling the definition of stable critical point presented in Sect. 2.2, we infer that for any $\varepsilon \approx 0$ the function $\frac{1}{2 \varepsilon}\left(E_{\varepsilon}\left(u_{q}^{\varepsilon}\right)-E_{0}\left(\mathrm{U}_{q}\right)\right)$ has a critical point $q^{\varepsilon} \in A$, to which corresponds the embedded $(k+\varepsilon \phi)$-bubble $u^{\varepsilon}:=u_{q^{\varepsilon}}^{\varepsilon}$ by iii) in Lemma 4.1. The continuity of $(\varepsilon, q) \mapsto u_{q}^{\varepsilon}$ gives the continuity of $\varepsilon \mapsto u^{\varepsilon}$.

The last conclusion in Theorem 1.2 follows via a simple compactness argument and thanks to Theorem 4.1 .

Proof of Theorem 1.3 Recalling that $q^{k}:=\left(q_{1}, q_{2}, k r_{k} q_{3}\right)$, we write

$$
F_{k}^{\phi}(q)=\int_{B_{r_{k}}(0)}\left(p_{3}+k r_{k}\right)^{-3} \phi\left(q_{3} p+q^{k}\right) d p .
$$

Since $r_{k} \rightarrow 0$ and $k r_{k}=k\left(k^{2}-1\right)^{-1 / 2} \rightarrow 1$ as $k \rightarrow \infty$, we infer that $q^{k} \rightarrow q$ uniformly on compact sets of $\mathbb{R}_{+}^{3}$ and

$$
\frac{3}{4 \pi r_{k}^{3}} F_{k}^{\phi} \rightarrow \phi \quad \text { as } k \rightarrow \infty
$$


uniformly on $\bar{\Omega}$. Next, we easily compute

$$
\begin{aligned}
& \partial_{q_{j}} F_{k}^{\phi}(q)=\int_{B_{r_{k}}(0)}\left(p_{3}+k r_{k}\right)^{-3} \partial_{q_{j}} \phi\left(q_{3} p+q^{k}\right) d p, \quad j=1,2, \\
& \partial_{q_{3}} F_{k}^{\phi}(q)=\int_{B_{r_{k}}(0)}\left(p_{3}+k r_{k}\right)^{-3} \nabla \phi\left(q_{3} p+q^{k}\right) \cdot\left(p+k r_{k} e_{3}\right) d p,
\end{aligned}
$$

and thus we obtain, by the same argument,

$$
\frac{3}{4 \pi r_{k}^{3}} \nabla F_{k}^{\phi} \rightarrow \nabla \phi \quad \text { as } k \rightarrow \infty,
$$

uniformly on $\bar{\Omega}$. It follows that for $k$ large enough, $F_{k}^{\phi}$ has a stable critical point in $\Omega \Subset \mathbb{H}^{3}$, since having a stable critical point is a $\mathcal{C}^{1}$-open condition. Hence Theorem 1.1 applies and gives the conclusion of the proof.

Acknowledgements This work is partially supported by PRID Projects PRIDEN and VAPROGE, Dipartimento di Scienze Matematiche, Informatiche e Fisiche, Università di Udine.

Funding Open access funding provided by Università degli Studi di Udine within the CRUI-CARE Agreement.

\section{Compliance with ethical standards}

Conflict of interest We declare there are no conflicts of interest/competing interests.

Open Access This article is licensed under a Creative Commons Attribution 4.0 International License, which permits use, sharing, adaptation, distribution and reproduction in any medium or format, as long as you give appropriate credit to the original author(s) and the source, provide a link to the Creative Commons licence, and indicate if changes were made. The images or other third party material in this article are included in the article's Creative Commons licence, unless indicated otherwise in a credit line to the material. If material is not included in the article's Creative Commons licence and your intended use is not permitted by statutory regulation or exceeds the permitted use, you will need to obtain permission directly from the copyright holder. To view a copy of this licence, visit http://creativecommons.org/licenses/by/4.0/.

\section{Appendix}

Let $K \in \mathcal{C}^{0}\left(\mathbb{H}^{3}\right)$. Take any vectorfield $Q_{K} \in \mathcal{C}^{1}\left(\mathbb{R}_{+}^{3}, \mathbb{R}^{3}\right)$ such that $\operatorname{div} Q_{K}(p)=p_{3}^{-3} K(p)$ for any $p \in \mathbb{R}_{+}^{3}$ (here div $=\sum_{j} \partial_{j}$ is the Euclidean divergence). The functional

$$
V_{K}(u):=\int_{\mathbb{R}^{2}} Q_{K}(u) \cdot \partial_{x} u \wedge \partial_{y} u d z, \quad u \in \mathcal{C}^{1}\left(\overline{\mathbb{R}}^{2}, \mathbb{H}^{3}\right),
$$

measures the signed (hyperbolic) volume enclosed by the surface $u$, with respect to the weight $K$. In fact, if $u$ parameterizes the boundary of a smooth open set $\Omega \Subset \mathbb{R}_{+}^{3}$ and if $\partial_{x} u \wedge \partial_{y} u$ is inward-pointing, then the divergence theorem gives

$$
V_{K}(u)=-\int_{\partial \Omega} Q_{K}(u) \cdot v d u=-\int_{\Omega} p_{3}^{-3} K d p=-\int_{\Omega} K d \mathbb{H}^{3} .
$$


Clearly, the functional $V_{K}$ does not depend on the choice of the vectorfield $Q$. Notice that if $K \equiv k$ is constant, then

$$
V_{k}(u)=-\frac{k}{2} \int_{\mathbb{R}^{2}} u_{3}^{-2} e_{3} \cdot \partial_{x} u \wedge \partial_{y} u d z, \quad u \in \mathcal{C}^{1}\left(\overline{\mathbb{R}}^{2}, \mathbb{H}^{3}\right) .
$$

In the next Lemma we collect few simple remarks about the energy functional

$$
E(u)=\frac{1}{2} \int_{\mathbb{R}^{2}} u_{3}^{-2}|\nabla u|^{2} d z+2 V_{K}(u) .
$$

Lemma A.1 Let $K \in \mathcal{C}^{0}\left(\mathbb{H}^{3}\right)$.

(i) The functional $E: \mathcal{C}^{1}\left(\overline{\mathbb{R}}^{2}, \mathbb{H}^{3}\right) \rightarrow \mathbb{R}$ is of class $\mathcal{C}^{1}$, and its differential is given by

$$
E^{\prime}(u) \varphi=\int_{\mathbb{R}^{2}}\left(u_{3}^{-2} \nabla u \cdot \nabla \varphi-u_{3}^{-3}|\nabla u|^{2} e_{3} \cdot \varphi\right) d z+2 \int_{\mathbb{R}^{2}} u_{3}^{-3} K(u) \varphi \cdot \partial_{x} u \wedge \partial_{y} u d z ;
$$

(ii) If $u \in \mathcal{C}^{2}\left(\overline{\mathbb{R}}^{2}, \mathbb{H}^{3}\right)$, then $E^{\prime}(u)$ extends to a continuous form on $\mathcal{C}^{0}\left(\overline{\mathbb{R}}^{2}, \mathbb{R}^{3}\right)$, namely

$$
E^{\prime}(u) \varphi=\int_{\mathbb{R}^{2}}\left(-\operatorname{div}\left(u_{3}^{-2} \nabla u\right)-u_{3}^{-3}|\nabla u|^{2} e_{3}+2 u_{3}^{-3} K(u) \partial_{x} u \wedge \partial_{y} u\right) \cdot \varphi d z ;
$$

(iii) If $K \in \mathcal{C}^{1}\left(\mathbb{H}^{3}\right)$, then $E$ is of class $\mathcal{C}^{2}$ on $\mathcal{C}^{1}\left(\overline{\mathbb{R}}^{2}, \mathbb{H}^{3}\right)$.

In the next Lemma we show that critical points for $E$ are in fact hyperbolic $K$-bubbles.

Lemma A.2 Let $K \in \mathcal{C}^{0}\left(\mathbb{H}^{3}\right)$ and let $u \in \mathcal{C}^{2}\left(\overline{\mathbb{R}}^{2}, \mathbb{H}^{3}\right)$ be a nonconstant critical point for $E$. Then $u$ is conformal, that is,

$$
\left|\partial_{x} u\right|=\left|\partial_{y} u\right|, \quad \partial_{x} u \cdot \partial_{y} u=0,
$$

hence it parameterizes an $\mathbb{S}^{2}$ type surface in $\mathbb{H}^{3}$, having mean curvature $K$, apart from a finite number of branch points.

Proof Put $\alpha=\frac{1}{2} u_{3}^{-2}\left(\left|\partial_{x} u\right|^{2}-\left|\partial_{y} u\right|^{2}\right), \beta=-u_{3}^{-2} \partial_{x} u \cdot \partial_{y} u, \varphi=\alpha+i \beta$ and notice that $|\varphi| \leq c_{u}|\nabla u|^{2} \in L^{\infty}\left(\mathbb{R}^{2}\right)$. By direct computation we find

$$
\begin{aligned}
& \left(\partial_{x} \alpha-\partial_{y} \beta\right) u_{3}^{3}=u_{3} \partial_{x} u \cdot \Delta u-\left(\left|\partial_{x} u\right|^{2}-\left|\partial_{y} u\right|^{2}\right) \partial_{x} u_{3}-2\left(\partial_{x} u \cdot \partial_{y} u\right) \partial_{y} u_{3}, \\
& \left(\partial_{y} \alpha+\partial_{x} \beta\right) u_{3}^{3}=-u_{3} \partial_{y} u \cdot \Delta u-\left(\left|\partial_{x} u\right|^{2}-\left|\partial_{y} u\right|^{2}\right) \partial_{y} u_{3}+2\left(\partial_{x} u \cdot \partial_{y} u\right) \partial_{x} u_{3} .
\end{aligned}
$$

Since $u$ solves (1.1), it holds that

$$
\begin{aligned}
& u_{3} \partial_{x} u \cdot \Delta u=2 G(\nabla u) \cdot \partial_{x} u=2\left(\partial_{x} u \cdot \partial_{y} u\right) \partial_{y} u_{3}+\left(\left|\partial_{x} u\right|^{2}-\left|\partial_{y} u\right|^{2}\right) \partial_{x} u_{3}, \\
& u_{3} \partial_{y} u \cdot \Delta u=2 G(\nabla u) \cdot \partial_{y} u=2\left(\partial_{x} u \cdot \partial_{y} u\right) \partial_{x} u_{3}-\left(\left|\partial_{x} u\right|^{2}-\left|\partial_{y} u\right|^{2}\right) \partial_{y} u_{3} .
\end{aligned}
$$

Putting together (A.2) and (A.3) we obtain $\partial_{x} \alpha-\partial_{y} \beta=\partial_{y} \alpha+\partial_{x} \beta=0$, namely, $\varphi$ is an holomorphic function. Since $\varphi$ is bounded and vanishes at infinity then $\varphi \equiv 0$ on $\mathbb{R}^{2}$, hence $u$ is conformal.

The last conclusion follows from Proposition 2.4 and Example 2.5(4) in [14]. 
Remark $A .1$ Here we take $K \equiv k$ constant and point out two simple facts about the energy functional $E_{\text {hyp }}$ in (1.3).

By (4.2), the Nehari manifold contains any nonconstant function. Secondly, $E_{\text {hyp }}$ is unbounded from below. In fact, for $t>1$ we have

$$
\begin{aligned}
E_{\text {hyp }}\left(\omega+t e_{3}\right) & =\frac{1}{2} \int_{\mathbb{R}^{2}}\left(\omega_{3}+t\right)^{-2} \mu^{2} d z+k \int_{\mathbb{R}^{2}}\left(\omega_{3}+t\right)^{-2} \omega_{3} \mu^{2} d z \\
& =4 \pi\left(-\frac{k t-1}{t^{2}-1}+\frac{k}{2} \ln \frac{t+1}{t-1}\right) .
\end{aligned}
$$

Notice that $\omega+t e_{3}$ approaches a horosphere as $t \rightarrow 1$, and that $\lim _{t \rightarrow 1} E_{\mathrm{hyp}}\left(\omega+t e_{3}\right)=-\infty$.

Remark A.2 Differently from the Euclidean case, see for instance [5], the geometric and compactness properties of the energy functional $E$ are far from being understood (also in the case of a constant curvature), and would deserve a careful analysis.

We conclude the paper by pointing out a necessary condition for the existence of embedded $K$ bubbles.

Let $K \in \mathcal{C}^{1}\left(\mathbb{H}^{3}\right)$ be given, and let $u \in \mathcal{C}^{2}\left(\overline{\mathbb{R}}^{2}, \mathbb{H}^{3}\right)$ be an embedded solution to (1.1). By Lemma A.2, $u$ is a conformal parametrization of the open set $\Omega \subset \mathbb{R}_{+}^{3}$, which is the bounded connected component of $\mathbb{R}_{+}^{3} \backslash u\left(\mathbb{S}^{2}\right)$. We can assume that the nowhere vanishing normal vector $\partial_{x} u \wedge \partial_{y} u$ is inward pointing. Since $u$ is a critical point of the energy functional in (A.1), then for $j=1,2$ we have that

$$
0=E^{\prime}(u) e_{j}=V_{K}^{\prime}(u) e_{j}=\int_{\mathbb{R}^{2}} u_{3}^{-3} K(u) e_{j} \cdot \partial_{x} u \wedge \partial_{y} u d z=-\int_{\Omega} \operatorname{div}\left(p_{3}^{-3} K(p) e_{j}\right) d p
$$

by the divergence theorem. Thus

$$
\int_{\Omega} p_{3}^{-3} \partial_{p_{j}} K(p) d p=0 .
$$

In a similar way, from $E^{\prime}(u) u=0$ and $\operatorname{since} \operatorname{div}\left(p_{3}^{-3} K(p) p\right)=p_{3}^{-3} \nabla K(p) \cdot p$, one gets

$$
\int_{\Omega} p_{3}^{-3} \nabla K(p) \cdot p d p=0
$$

In particular, $\partial_{p_{1}} K, \partial_{p_{2}} K$ and the radial derivative of $K$ can not have constant sign in $\Omega$. We infer the next nonexistence result (see [7, Proposition 4.1] for the Euclidean case).

Theorem A.3 Assume that $K \in \mathcal{C}^{1}\left(\mathbb{H}^{3}\right)$ satisfies one of the following conditions,

(i) $K(p)=f(v \cdot p)$ for some direction $v$ orthogonal $e_{3}$, where $f$ is strictly monotone;

(ii) $K(p)=f(|p|)$, where $f$ is strictly monotone.

Then (1.1) has no embedded solution $u \in \mathcal{C}^{2}\left(\overline{\mathbb{R}}^{2}, \mathbb{H}^{3}\right)$.

\section{References}

1. Alencar, H., Rosenberg, H.: Some remarks on the existence of hypersurfaces of constant mean curvature with a given boundary, or asymptotic boundary, in hyperbolic space. Bull. Sci. Math. 121(1), 61-69 (1997) 
2. Ambrosetti, A., Malchiodi, A.: Perturbation Methods and Semilinear Elliptic Problems on $\mathbb{R}^{n}$, Progress in Mathematics, 240. Birkhäuser Verlag, Basel (2006)

3. Anderson, M.T.: Conformal immersions of prescribed mean curvature in $\mathbb{R}^{3}$. Nonlinear Anal. 114, 142157 (2015)

4. Aubin, T.: Nonlinear Analysis on Manifolds. Monge-Ampère Equations, Grundlehren der Mathematischen Wissenschaften, 252. Springer, New York (1982)

5. Brezis, H., Coron, J.-M.: Convergence of solutions of $H$-systems or how to blow bubbles. Arch. Ration. Mech. Anal. 89(1), 21-56 (1985)

6. Caldiroli, P., Cora, G.: On the dynamics of a charged particle in magnetic fields with cylindrical symmetry. J. Differ. Equ. 267(6), 3952-3976 (2019)

7. Caldiroli, P., Musina, R.: $H$-bubbles in a perturbative setting: the finite-dimensional reduction method. Duke Math. J. 122(3), 457-484 (2004)

8. Caldiroli, P., Musina, R.: The Dirichlet problem for $H$-systems with small boundary data: blowup phenomena and nonexistence results. Arch. Ration. Mech. Anal. 181(1), 1-42 (2006)

9. Caldiroli, P., Musina, R.: Weak limit and blowup of approximate solutions to $H$-systems. J. Funct. Anal. 249(1), 171-198 (2007)

10. Caldiroli, P., Musina, R.: Bubbles with prescribed mean curvature: the variational approach. Nonlinear Anal. 74(9), 2985-2999 (2011)

11. Chanillo, S., Malchiodi, A.: Asymptotic Morse theory for the equation $\Delta v=2 v_{x} \wedge v_{y}$. Comm. Anal. Geom. 13(1), 187-251 (2005)

12. Felli, V.: A note on the existence of $H$-bubbles via perturbation methods. Rev. Mat. Iberoamericana 21(1), 163-178 (2005)

13. Gulliver, R.D., II.: The Plateau problem for surfaces of prescribed mean curvature in a Riemannian manifold. J. Differ. Geom. 8, 317-330 (1973)

14. Gulliver, R.D., II., Osserman, R., Royden, H.L.: A theory of branched immersions of surfaces. Am. J. Math. 95, 750-812 (1973)

15. Isobe, T.: On the asymptotic analysis of $H$-systems. I. Asymptotic behavior of large solutions. Adv. Differ. Equ. 6(5), 513-546 (2001)

16. López, R.: Constant Mean Curvature Surfaces with boundary, Springer Monographs in Mathematics. Springer, Heidelberg (2013)

17. Musina, R.: The role of the spectrum of the Laplace operator on $\mathbb{S}^{2}$ in the $\mathcal{H}$-bubble problem. J. Anal. Math. 94, 265-291 (2004)

18. Musina, R., Zuddas, F.: Embedded loops in the hyperbolic plane with prescribed, almost constant curvature. Ann. Global Anal. Geom. 55(3), 509-528 (2019)

19. Nardulli, S.: The isoperimetric profile of a smooth Riemannian manifold for small volumes. Ann. Global Anal. Geom. 36(2), 111-131 (2009)

20. Treibergs, A.E., Wei, S.W.: Embedded hyperspheres with prescribed mean curvature. J. Differ. Geom. 18(3), 513-521 (1983)

21. Yau, S.T.: A remark on the existence of sphere with prescribed mean curvature. Asian J. Math. 1(2), 293-294 (1997)

22. Yau, S..T.: Problem section. In: Yau, S.-T. (ed.) Seminar on Differential Geometry, Annals of Mathematical Studies, vol. 102, pp. 669-706. Princeton Univ. Press, Princeton, NJ (1982)

23. Ye, R.: Foliation by constant mean curvature spheres. Pac. J. Math. 147(2), 381-396 (1991)

Publisher's Note Springer Nature remains neutral with regard to jurisdictional claims in published maps and institutional affiliations. 